\title{
Analysis of Jet Transport-based Geostationary Trajectory Uncertainty Propagation
}

\author{
Jianlin Chen * \\ National Key Laboratory of Aerospace Flight Dynamics \& Northwestern Polytechical University \& \\ Universitat Politècnica de Catalunya \\ Josep J. Masdemont ${ }^{\dagger}$ \\ IEEC \& Universitat Politècnica de Catalunya \\ Gerard Gómez \\ IEEC \& Universitat de Barcelona \\ Jianping Yuan $\S$ \\ National Key Laboratory of Aerospace Flight Dynamics \& Northwestern Polytechical University
}

This paper investigates an accurate and efficient nonlinear method for propagating geostationary trajectory uncertainties under four dominant perturbations: solar radiation pressure, Earth's non-spherical gravity potential, and luni-solar gravitational attractions. A Jet Transport-based scheme (also known as Differential Algebra) is considered and implemented in several coordinate representations, and in two forms: Taylor and Chebyshev expansions. Taking into account the size of the uncertainty neighborhood, as well as the order and time steps of the integration schemes, a large amount of combinations of the above factors are simulated, and a series of look-up tables with recommendations on the best options to address the propagations are given.

\section{Nomenclature}

$$
\begin{array}{ll}
\boldsymbol{a}_{c}, \boldsymbol{a}_{G} & =\text { perturbing accelerations in Cartesian coordinates and GEO elements, respectively } \\
\boldsymbol{a}_{k_{1} \ldots k_{n}} & =\text { Taylor expansion coefficients } \\
A & =\text { cross-section area } \\
A_{n} & =\text { nominal GEO semi-major axis } \\
\boldsymbol{A} & =\text { derivatives of GEO elements with respect to Cartesian coordinates } \\
\boldsymbol{b}_{k_{1} \ldots k_{n}} & =\text { Chebyshev expansion coefficients } \\
\boldsymbol{B} & =\text { derivatives of GEO elements with respect to Earth-fixed sub-spacecraft longitude }
\end{array}
$$

\footnotetext{
*Ph.D. School of Astronautics, Road West 127, Xi'an 710072, China. chenj1@mail.nwpu.edu.cn.

†Professor. UPC-Departament de Matemàtiques, Diagonal 647, 08028 Barcelona. josep.masdemont@ upc.edu.

†Professor. UB-Departament de Matemàtiques i Informàtica, Gran Via de les Corts Catalanes 585, 08007 Barcelona. gerard@maia.ub.es.

§Professor. School of Astronautics, Road West 127, Xi'an 710072, China. jyuan@nwpu.edu.cn.
} 


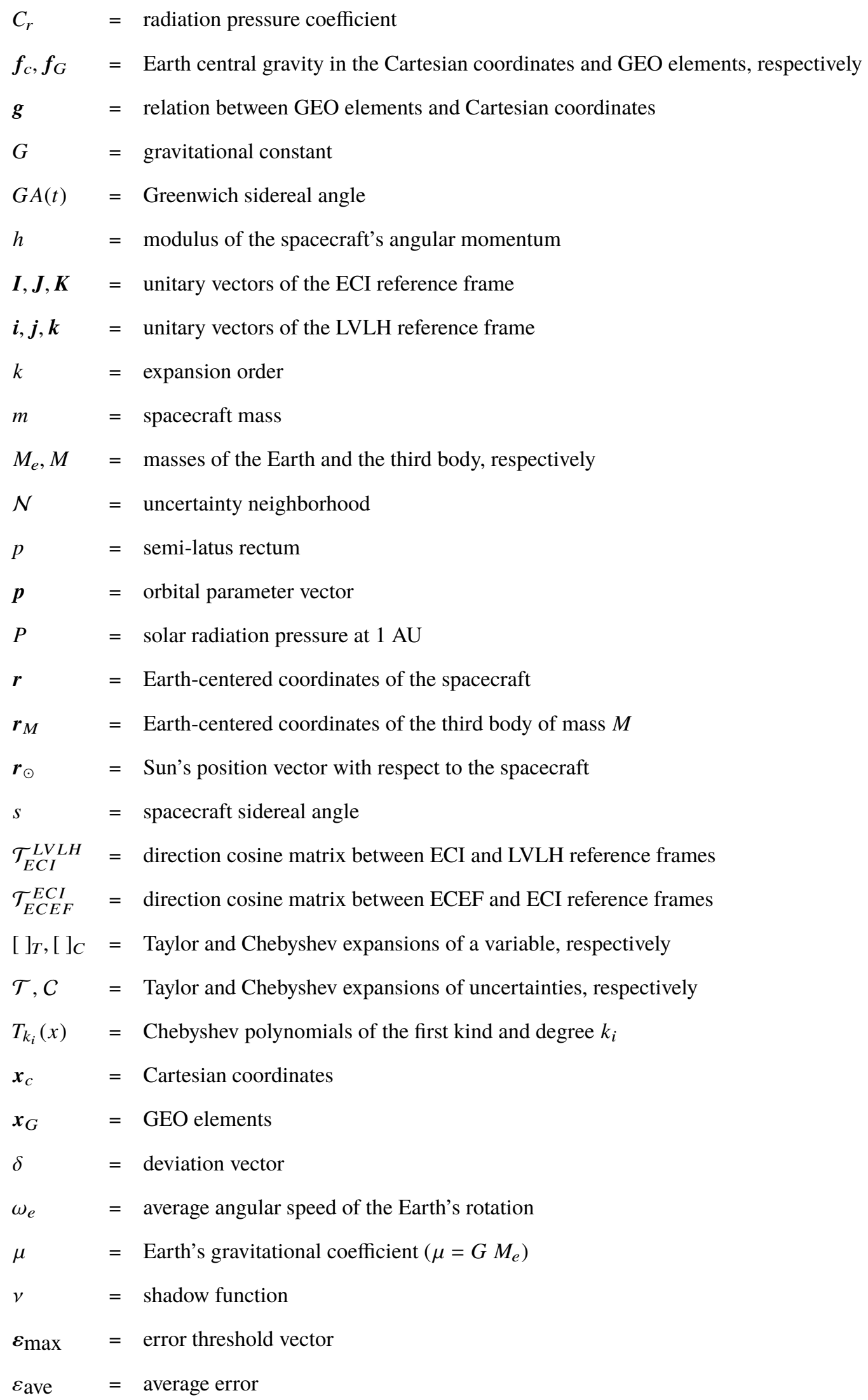


$\begin{array}{ll}\sigma & =\text { variance } \\ \boldsymbol{\Phi} & =\text { differential equation flow }\end{array}$

\section{Introduction}

$\mathrm{T}$ HE Geostationary Earth Orbit (GEO) regime provides a special type of orbits that have the same period as the rotation period of the Earth and, therefore, maintain in a nearly-constant position in an Earth-centered Earth-fixed (ECEF) reference frame. Due to this feature, GEO orbits are beneficial to perform continuous observation of fixed regions of the Earth's surface, as well as to provide direct broadcast and communication services. However, the increasing amount of geostationary objects, involving controlled GEO spacecraft and uncontrolled debris, aggravate the congestion of this high valued region increasing the collision risks. To monitor geostationary objects, a regular report supported by ESA's Database and Informations System Characterizing Objects in Space is available. According to Lemmens [U] , the latest report reveals a detailed information of 1533 unclassified objects in or near the GEO region, from which only 502 are satellites capable of performing active orbit and attitude control. To avoid collisions, the concept of Region B has been recently defined [2] with the purpose of restricting the number of geostationary spacecraft inside a specific spherical shell (GEO altitude $\pm 200 \mathrm{~km}$ and equatorial latitude \pm 15 degrees). Therefore, it is a general practice for geostationary spacecraft operators to compute the accurate propagation of geostationary trajectories and maintain them in a specified region.

Nevertheless, a high accurate and efficient orbit propagation and determination for geostationary satellites is not only justified for Monte Carlo analysis, collision avoidance or efficient station keeping. New emerging applications of GEO satellites are considered. Future GeoSAR missions monitoring land stability subjected to natural hazards like volcanic activity or earthquakes will need for an unprecedent accuracy [3]. This accuracy is also required for mezzoscale measurements in sea altimetry using TV signals from GEO satellites which are stronger and more convenient than the GPS ones [4]].

Several types of coordinate representations have been investigated to describe the evolution of geostationary trajectories. They are summarized in detail in [5, 6]. The traditional equations of motion for orbit determination and propagation, by means of inertial position and velocity vectors, are capable of providing physical insight into the dynamics; however, the fast changes of these coordinates over time, obscure the beneficial characteristic that geostationary spacecrafts remain almost steady in the ECEF frame. Keplerian orbital elements have this advantage and mitigate numerical issues due to small integration time step sizes but, unfortunately, they are not well suited for the geotrajectory orbit propagation, due to their poor definitions of low-inclination or low-eccentricity orbits. To avoid these inconveniences, a synchronous orbit element set was introduced by Soop [5], and later, the dynamical model in these new coordinates was developed by Tombasco [6, 7]. However, this model still has numerical sensitivity issues 
for very small inclination angles and, in these papers, the GEO orbit element set was defined in terms of the classical Keplerian elements, developing as well the resulting nonlinear variational equations for dominant perturbations in the GEO regime. The resulting model called as GEO dynamic model and described in section III, plays a major role in the discussions of our work.

From another side, linearized propagation models [8] are useful for the propagation of spacecraft trajectories and parameter uncertainties, despite their poor accuracy, or even failure evolution, in cases with strong nonlinear dynamical behavior. To overcome this drawback and to describe the statistics of the uncertainties, the usual alternative is to do nonlinear Monte Carlo simulations [9]; however, their computational cost is huge due to the poor convergence of Monte Carlo statistics, associated just to a particular epoch, which limits its applicability.

In 2006, Park and Scheeres [10] investigated the Fokker-Planck equation solutions showing that the nonlinear propagation of trajectory uncertainties can be explicitly given by a high order Taylor series expansion. They proved that the results provided by their method are close to the ones given by means of Monte Carlo simulations. However, the computation of the coefficients of the high order Taylor expansion is computationally intensive and expensive.

The main purpose of this paper is the efficient, simple, and precise determination, using high-order expansions, of the evolution of trajectories and parameter uncertainties in the GEO regime by means of the Jet Transport (JT) propagation technique (described in section III). The questions considered include: the performance comparison of Taylor and Chebyshev expansions; the determination of the nonlinear transformation between GEO elements and Cartesian coordinates; the expansion order analysis of the dominant perturbations; and the performance analysis of JT-based GEO trajectory uncertainties propagation.

As a summary, this paper provides a new accurate strategy for the propagation of GEO trajectories and parameter uncertainties, that opens the possibilities of the use of GEOs to some new applications, as the ones described at the beginning of this Introduction.

\section{Dynamical models}

Several coordinate representations of spacecraft trajectories have been extensively studied, and a variety of dynamic models have been proposed. Cartesian coordinates can be used to easily model the dominant perturbations in the GEO region, including solar radiation pressure (SRP), Earth's non-spherical gravity, or luni-solar gravitational attraction, but their fast variation highlights the shortcomings, such as small integration steps and high computational costs. Due to this reason other element sets are used and they are discussed in what follows. 


\section{Cartesian dynamic model}

The motion of a spacecraft near the Earth is governed by a set of three second order ordinary differential equations (ODEs) that, as it is well known, in a Cartesian inertial frame are:

$$
\begin{aligned}
& \ddot{x}=-\frac{\mu x}{r^{3}}+a_{x}, \\
& \ddot{y}=-\frac{\mu y}{r^{3}}+a_{y}, \\
& \ddot{z}=-\frac{\mu z}{r^{3}}+a_{z},
\end{aligned}
$$

where, in an Earth-Centered Inertial reference frame (ECI), $\boldsymbol{a}=\left(a_{x}, a_{y}, a_{z}\right)^{T}$ is the perturbing acceleration, $\boldsymbol{r}=$ $(x, y, z)^{T}$ is the spacecraft position, and $\mu=G M_{e}$ is the gravitational coefficient of the Earth. We can also write these equations in a compact form as,

$$
\dot{\boldsymbol{x}}_{c}=\boldsymbol{f}_{c}\left(\boldsymbol{x}_{c}\right)+\boldsymbol{a}_{c}\left(\boldsymbol{x}_{c}, t\right)
$$

where $\boldsymbol{x}_{c}=\{x, y, z, \dot{\boldsymbol{x}}, \dot{y}, \dot{z}\}$, and the functions $\boldsymbol{f}_{c}\left(\boldsymbol{x}_{c}\right)$ and $\boldsymbol{a}_{c}\left(\boldsymbol{x}_{c}, t\right)$ denote, respectively, the Earth's central gravitational force and the perturbing accelerations acting on the spacecraft, this is,

$$
\begin{gathered}
\boldsymbol{f}_{c}\left(\boldsymbol{x}_{c}\right)=\left(\begin{array}{llllll}
\dot{x} & \dot{y} & \dot{z} & \frac{\mu}{r^{3}} x & \frac{\mu}{r^{3}} y & \frac{\mu}{r^{3}} z
\end{array}\right)^{T}, \\
\boldsymbol{a}_{c}\left(\boldsymbol{x}_{c}, t\right)=\left(\begin{array}{llllll}
0 & 0 & 0 & a_{x} & a_{y} & a_{z}
\end{array}\right)^{T} .
\end{gathered}
$$

\section{GEO dynamical model}

In [7] Tombasco introduced a novel non-dimensional GEO element set in terms of the classical Keplerian elements. It consists of the Earth-fixed sub-spacecraft longitude $\lambda$, the longitudinal drift rate $\delta \bar{a}$, two eccentricity vector components $\left(e_{x}, e_{y}\right)$, and two equinoctial elements $\left(Q_{1}, Q_{2}\right)$ defined by:

$$
\begin{aligned}
& \lambda \triangleq(\omega+\Omega+\theta)-G A(t) \\
& \delta \bar{a} \triangleq \frac{a-A_{n}}{A_{n}} \\
& e_{x} \triangleq e \cos (\omega+\Omega) \\
& e_{y} \triangleq e \sin (\omega+\Omega) \\
& Q_{1} \triangleq \tan \left(\frac{i}{2}\right) \sin (\Omega) \\
& Q_{2} \triangleq \tan \left(\frac{i}{2}\right) \cos (\Omega)
\end{aligned}
$$


here, $\{a, e, i, \omega, \Omega, \theta\}$ refer to the classical set of Keplerian elements, $\delta \bar{a}$ is non-dimensional value of the semi-major axis deviation with respect to a nominal GEO semi-major axis $A_{n}=42164.2 \mathrm{~km}$, and the time dependent function $G A(t)$ stands for the Greenwich sidereal angle at $t$, this is, $G A(t)=G A\left(t_{0}\right)+\omega_{e}\left(t-t_{0}\right)$, where $\omega_{e}=7.292115 \times 10^{-5} \mathrm{rad} / \mathrm{s}$ is the average angular speed of the Earth's rotation. Using the Poisson brackets method, Tombasco obtained in [7] the GEO dynamic model in terms of the GEO element set, that can be written as:

$$
\begin{aligned}
\dot{\lambda} & =\frac{h}{r^{2}}+\frac{r}{h}\left[Q_{2} \sin s-Q_{1} \cos s\right] a_{h}-\omega_{e}, \\
\delta \dot{\bar{a}} & =\frac{2(\delta \bar{a}+1)^{2}}{h A_{n}}\left[\left(e_{x} \sin s-e_{y} \cos s\right) a_{r}+\frac{p}{r} a_{\theta}\right], \\
\dot{e}_{x} & =\frac{r}{h}\left\{\frac{p}{r} \sin s \cdot a_{r}+\left[e_{x}+\left(1+\frac{p}{r}\right) \cos s\right] a_{\theta}+e_{y}\left[Q_{1} \cos s-Q_{2} \sin s\right] a_{h}\right\}, \\
\dot{e}_{y} & =\frac{r}{h}\left\{-\frac{p}{r} \cos s \cdot a_{r}+\left[e_{y}+\left(1+\frac{p}{r}\right) \sin s\right] a_{\theta}-e_{x}\left[Q_{1} \cos s-Q_{2} \sin s\right] a_{h}\right\}, \\
\dot{Q}_{1} & =\frac{r}{2 h}\left(1+Q_{1}^{2}+Q_{2}^{2}\right) \sin s \cdot a_{h}, \\
\dot{Q}_{2} & =\frac{r}{2 h}\left(1+Q_{1}^{2}+Q_{2}^{2}\right) \cos s \cdot a_{h},
\end{aligned}
$$

where the components of the perturbing acceleration $\boldsymbol{a}=\left(a_{r}, a_{\theta}, a_{h}\right)$ are given in the Local Vertical Local Horizontal reference frame (LVLH), $r$ is the radial distance of the spacecraft to the center of the Earth, $s$ is the spacecraft sidereal angle, $p$ is the semi-latus rectum of the orbit, and $h$ is the modulus of the angular momentum. Using the definitions of $r, s, p$, and $h$, they can be written in terms of GEO elements as:

$$
\begin{aligned}
& r=\frac{A_{n}(\delta \bar{a}+1)\left(1-e_{x}^{2}-e_{y}^{2}\right)}{1+e_{x} \cos s+e_{y} \sin s}, \\
& s=\lambda+G A(t)=\omega+\Omega+\theta, \\
& p=A_{n}(\delta \bar{a}+1)\left(1-e_{x}^{2}-e_{y}^{2}\right), \\
& h=\sqrt{p \mu} .
\end{aligned}
$$

In principle, the perturbing acceleration $\boldsymbol{a}$ in (6) can be easily determined using the spacecraft's Cartesian position but, in order to propagate trajectories in this set of differential equations, $\boldsymbol{a}$ must be computed as a function of the GEO elements. The procedure that has been used for the conversion is the following: at a certain epoch we transform the GEO elements of the spacecraft into Cartesian coordinates, then we compute the perturbing acceleration, and finally we project it into the LVLH reference frame. This involves the transformation from GEO elements to Cartesian coordinates, and the computation of the transformation matrix between the ECI and the LVLH reference frames. The numerical integrator makes an intensive use of this procedure to propagate a state in the GEO model.

Using the classical Keplerian element set as a bridge, we can obtain the explicit relationship between the GEO 
elements and the Cartesian coordinates (a detailed derivation is given in the Appendix). This is given by:

$$
\begin{aligned}
x & =\frac{r}{1+Q_{1}^{2}+Q_{2}^{2}}\left[2 Q_{1} Q_{2} \sin s+\left(1-Q_{1}^{2}+Q_{2}^{2}\right) \cos s\right], \\
y & =\frac{r}{1+Q_{1}^{2}+Q_{2}^{2}}\left[2 Q_{1} Q_{2} \cos s+\left(1+Q_{1}^{2}-Q_{2}^{2}\right) \sin s\right], \\
z & =\frac{2 r}{1+Q_{1}^{2}+Q_{2}^{2}}\left[Q_{2} \sin s-Q_{1} \cos s\right], \\
\dot{x} & =\frac{\mu}{h\left(1+Q_{1}^{2}+Q_{2}^{2}\right)}\left[2 Q_{1} Q_{2}\left(\cos s+e_{x}\right)+\left(-1+Q_{1}^{2}-Q_{2}^{2}\right)\left(\sin s+e_{y}\right)\right], \\
\dot{y} & =\frac{\mu}{h\left(1+Q_{1}^{2}+Q_{2}^{2}\right)}\left[-2 Q_{1} Q_{2}\left(\sin s+e_{y}\right)+\left(1+Q_{1}^{2}-Q_{2}^{2}\right)\left(\cos s+e_{x}\right)\right], \\
\dot{z} & =\frac{\mu}{h\left(1+Q_{1}^{2}+Q_{2}^{2}\right)}\left[Q_{1}\left(\sin s+e_{y}\right)+Q_{2}\left(\cos s+e_{x}\right)\right] .
\end{aligned}
$$

To determine the components of the the perturbing acceleration $\boldsymbol{a}$ in the LVLH reference frame, we need to compute the direction cosines matrix between the ECI and the LVLH reference frames, this is:

$$
\mathcal{T}_{E C I}^{L V L H}=\left(\begin{array}{ccc}
I \cdot i & I \cdot j & I \cdot k \\
J \cdot i & J \cdot j & J \cdot k \\
K \cdot i & K \cdot j & K \cdot k
\end{array}\right)
$$

where $\boldsymbol{I}, \boldsymbol{J}, \boldsymbol{K}$ are the unitary vectors defining the ECI reference frame, while $\boldsymbol{i}, \boldsymbol{j}, \boldsymbol{k}$ are the unitary vectors defining the LVLH frame, which can be computed as:

$$
i=\frac{r}{\|r\|}, \quad k=\frac{r \times v}{\|r \times v\|}, \quad j=k \times i
$$

Besides this explicit GEO model, another implicit form of the GEO element representation model has been obtained and implemented. For this purpose we rewrite the explicit relationship (II) between Cartesian coordinates and GEO elements as:

$$
\boldsymbol{x}_{c}=\boldsymbol{g}\left(\boldsymbol{x}_{G}, t\right)
$$

where $\boldsymbol{x}_{c}, \boldsymbol{x}_{G}$ respectively represent the Cartesian coordinates and the GEO elements. Derivating ([13) with respect to the time $t$ gives:

$$
\dot{\boldsymbol{x}}_{c}=\boldsymbol{A}\left(\boldsymbol{x}_{G}, t\right) \dot{\boldsymbol{x}}_{G}+\boldsymbol{B}\left(\boldsymbol{x}_{G}, t\right)
$$


where the matrix $\boldsymbol{A}\left(\boldsymbol{x}_{G}, t\right)$, and the vector $\boldsymbol{B}\left(\boldsymbol{x}_{G}, t\right)$ are:

$$
\boldsymbol{A}\left(\boldsymbol{x}_{G}, t\right)=\frac{\partial \boldsymbol{g}}{\partial \boldsymbol{x}_{G}}=\left(\begin{array}{llllll}
\frac{\partial g_{1}}{\partial \lambda} & \frac{\partial g_{1}}{\partial \delta \bar{a}} & \frac{\partial g_{1}}{\partial e_{x}} & \frac{\partial g_{1}}{\partial e_{y}} & \frac{\partial g_{1}}{\partial Q_{1}} & \frac{\partial g_{1}}{\partial Q_{2}} \\
\frac{\partial g_{2}}{\partial \lambda} & \frac{\partial g_{2}}{\partial \delta \bar{a}} & \frac{\partial g_{2}}{\partial e_{x}} & \frac{\partial g_{2}}{\partial e_{y}} & \frac{\partial g_{2}}{\partial Q_{1}} & \frac{\partial g_{2}}{\partial Q_{2}} \\
\frac{\partial g_{3}}{\partial \lambda} & \frac{\partial g_{3}}{\partial \delta \bar{a}} & \frac{\partial g_{3}}{\partial e_{x}} & \frac{\partial g_{3}}{\partial e_{y}} & \frac{\partial g_{3}}{\partial Q_{1}} & \frac{\partial g_{3}}{\partial Q_{2}} \\
\frac{\partial g_{4}}{\partial \lambda} & \frac{\partial g_{4}}{\partial \delta \bar{a}} & \frac{\partial g_{4}}{\partial e_{x}} & \frac{\partial g_{4}}{\partial e_{y}} & \frac{\partial g_{4}}{\partial Q_{1}} & \frac{\partial g_{4}}{\partial Q_{2}} \\
\frac{\partial g_{5}}{\partial \lambda} & \frac{\partial g_{5}}{\partial \delta \bar{a}} & \frac{\partial g_{5}}{\partial e_{x}} & \frac{\partial g_{5}}{\partial e_{y}} & \frac{\partial g_{5}}{\partial Q_{1}} & \frac{\partial g_{5}}{\partial Q_{2}} \\
\frac{\partial g_{6}}{\partial \lambda} & \frac{\partial g_{6}}{\partial \delta \bar{a}} & \frac{\partial g_{6}}{\partial e_{x}} & \frac{\partial g_{6}}{\partial e_{y}} & \frac{\partial g_{6}}{\partial Q_{1}} & \frac{\partial g_{6}}{\partial Q_{2}}
\end{array}\right), \quad \boldsymbol{B}\left(\boldsymbol{x}_{G}, t\right)=\frac{\partial \boldsymbol{g}}{\partial t}=\omega_{e}\left(\begin{array}{c}
\frac{\partial g_{1}}{\partial \lambda} \\
\frac{\partial g_{2}}{\partial \lambda} \\
\frac{\partial g_{3}}{\partial \lambda} \\
\frac{\partial g_{4}}{\partial \lambda} \\
\frac{\partial g_{5}}{\partial \lambda} \\
\frac{\partial g_{6}}{\partial \lambda}
\end{array}\right)
$$

The components of both $\boldsymbol{A}\left(\boldsymbol{x}_{G}, t\right)$ and $\boldsymbol{B}\left(\boldsymbol{x}_{G}, t\right)$ can be explicitly computed and their expressions are given in the Appendix. Substituting ([1]) into ([2) we obtain:

$$
\dot{x}_{c}=f_{G}\left(x_{G}\right)+a_{G}\left(x_{G}, t\right)
$$

where $f_{G}\left(x_{G}\right)$ and $a_{G}\left(x_{G}, t\right)$ respectively represent the Earth's central gravity and the general perturbing accelerations

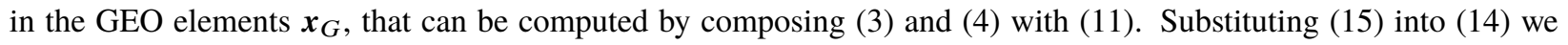
obtain an exact implicit version of the nonlinear equations of motion:

$$
\dot{\boldsymbol{x}}_{G}=\boldsymbol{A}^{-1}\left(f_{G}+a_{G}-B\right)
$$

where $\boldsymbol{A}^{-1}$ can be calculated automatically and efficiently using the JT technique to be discussed in the next section. It should be noted that the derivation of (116) is a straightforward computation, and is also a general method for rewriting vector fields in different coordinates.

We note that the models defined by (6) and (106) are two different sets of equations, explicit and implicit, of the same GEO model. We have validated that their accuracies are of the same order of magnitude. The main difference between them resides in the orbit propagation computational cost, whose performance a priori is not obvious due to the changes of coordinates involved when considering perturbations. For instance, when compared to (河), the implementation of (ㅁ) eliminates the additional conversion of the perturbing accelerations from the ECI frame to the LVLH frame, and it expresses the perturbing accelerations directly along the axes of the ECI frame. From this point of view, it might seem that the computational cost of (ㅁ) could be less than the one of (6). However, the simulations have shown that the computational time required to propagate in ([16) is a little bit larger due to the calculation of the inverse of $\boldsymbol{A}$. Therefore, when considering the GEO model, the explicit form (6) has been the one adopted in this manuscript. 


\section{Jet Transport}

Jet Transport (JT), also known as Differential Algebra (DA), is essentially an automatic differentiation technique that provides the Taylor or Chebyshev expansion, up to an arbitrary order, of the flow associated to an ODE. The applications of the JT technique, as a means of symbolic computation, have received an increasing attention in recent years. Even that the main idea of using Taylor, or other kind of expansions, as formal solutions is a classical procedure in Dynamical Systems and Celestial Mechanics, the JT formalization was first done in 1999 by Berz and Makino [П]] for the study of particle beam accelerators, and implemented by the same authors in their COSY Infinity package.

Subsequently, several researchers focused on exploring this methodology in a number of different space applications, such as the propagation of trajectory uncertainties for low Earth orbits [12], relative state estimation [1]3], orbit determination [14, 15], or propagation features with autonomous and non-autonomous perturbations [16]. See also [177] and references therein for more applications.

Up to date, JT-based applications extensively use Taylor series to expand nonlinear functions around a certain point. However, Taylor polynomial accuracy drops off for highly nonlinear dynamics, large neighborhoods around the expansion point, and long-term propagations. To address these problems, automatic domain splitting techniques have been developed [18, 19]. In order to better "distribute" (min-max approximations) the error of the polynomial approximation in a certain range of interest, one can consider the use of Chebyshev polynomial approximations instead of Taylor expansions [20]. The implementation of JT using both Taylor and Chebyshev series was done by Ortega et al. [21] in the SMART-UQ package. For the GEO trajectory propagation under consideration, both approximations are compared in this work.

\section{JT-based nonlinear mapping}

The JT technique is capable of producing high order expansions of nonlinear functions, either in Taylor or Chebyshev series, in a systematic way. For the specific details about the involved algebra and computer implementation we refer to [II, [17, 21, 22], here we just provide the main ideas needed for the purposes of this paper.

Consider an arbitrary nonlinear transformation,

$$
y=f(x)
$$

where $\boldsymbol{x}$ indicates a vector consisting of $n$-dimensional independent variables that will be affected by some uncertainties. The goal is to map a neighborhood of $\boldsymbol{x}$ into a neighborhood of $\boldsymbol{y}$ by means of a simple but accurate representation. The JT implementations solve this problem for all the basic algebraic operations, elementary functions, composition, inverse of functions, etc. Then, the function $f$ can be the result of a very complex set of computations, for instance, even we can think of $\boldsymbol{x}$ being an initial state and $\boldsymbol{y}$ the result of the propagation of this initial state in a complex nonlinear 
vectorfield.

The procedure can be summarized as follows. First, the independent variable vector $\boldsymbol{x}$ is defined as a JT-based vector $[\boldsymbol{x}]=\overline{\boldsymbol{x}}+\delta \boldsymbol{x}$, that is, the sum of the nominal state, or initial mean $\overline{\boldsymbol{x}}$, and some undetermined deviation $\delta \boldsymbol{x}$. Then, the output of (ID7) in the JT scheme is a certain (truncated) expansion in terms of $\delta \boldsymbol{x}$. In our computations both Taylor and Chebyshev series are considered. For the Taylor series case, the resulting $k$ th-order expansion, as a truncated power series of deviation vector $\delta \boldsymbol{x}$, is:

$$
[\boldsymbol{y}]_{T}^{k}=\boldsymbol{f}([\boldsymbol{x}])_{\mid k}=\overline{\boldsymbol{y}}+\delta \boldsymbol{y}^{k}=\overline{\boldsymbol{y}}+\mathcal{T}_{\boldsymbol{y}}^{k}(\delta \boldsymbol{x})=\sum_{0 \leq k_{1}+\cdots+k_{n} \leq k} \boldsymbol{a}_{k_{1} \ldots k_{n}} \delta x_{1}^{k_{1}} \ldots \delta x_{n}^{k_{n}}
$$

where, from now on, [ ] $]_{T}^{k}$ indicates the Taylor expansion for $\boldsymbol{y}$ up to order $k, \delta \boldsymbol{y}=\mathcal{T}_{\boldsymbol{y}}{ }^{k}$, represents the expansion for the uncertainty $\delta \boldsymbol{y}$ in terms of $\delta \boldsymbol{x}$ using Taylor series, while $\overline{\boldsymbol{y}}$ is the zeroth-order term, and $\boldsymbol{a}_{k_{1} \ldots k_{n}}$ are the resulting Taylor coefficients. A similar implementation is performed using a set of orthonormal Chebyshev basis, which produces a $k$ th-order Chebyshev expansion relative to the deviation vector $\delta \boldsymbol{x}$ that will be written as:

$$
[\boldsymbol{y}]_{C}^{k}=\boldsymbol{f}([\boldsymbol{x}])=\overline{\boldsymbol{y}}+\delta \boldsymbol{y}^{k}=\overline{\boldsymbol{y}}+C_{\boldsymbol{y}}^{k}(\delta \boldsymbol{x})=\sum_{0 \leq k_{1}+\cdots+k_{n} \leq k} \boldsymbol{b}_{k_{1} \ldots k_{n}} T_{k_{1}}\left(\delta x_{1}\right) \ldots T_{k_{n}}\left(\delta x_{n}\right)
$$

where now [ $]_{C}^{k}$ indicates the Chebyshev expansion for $\boldsymbol{y}$ up to order $k, \delta \boldsymbol{y}=C_{\boldsymbol{y}}^{k}$ the Chebyshev expansion of the uncertainty $\delta \boldsymbol{y}, \overline{\boldsymbol{y}}$ the zeroth-order term, $\boldsymbol{b}_{k_{1} \ldots k_{n}}$ the coefficients of the Chebyshev expansion, and $T_{k_{i}}$ denote Chebyshev polynomials of the first kind and degree $k_{i}$. Here, it is worth to mention that, unlike the Taylor case, in the Chebyshev algebra the uncertainty region for $\delta \boldsymbol{x}$ has to be defined a priori. In this case $\delta \boldsymbol{x}=\delta x_{1} \times \delta x_{2} \times \cdots \times \delta x_{n}$ is an hypercube with $\delta x_{i}=[-\delta, \delta]$, and the $T_{k_{i}}$ family is the orthogonal one in this interval.

The expressions ([8) and (피) enable to map, in a simple and efficient way, the uncertainties $\delta \boldsymbol{x}$ to the solution $\boldsymbol{y}$ by means of an arbitrary $k$ th-order expansion. It is clear that the accuracy of this JT-based nonlinear map is affected

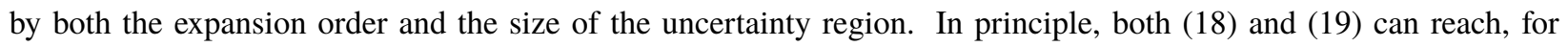
a given uncertainty region, the desired accuracy by adjusting the expansion orders. Besides, if $k=1$, they become the linear map approximation (i.e., the linearized model). In the case of orbit propagation, the usual state transition matrix is given by the coefficients $\boldsymbol{a}_{k_{1} \ldots k_{n}}$ or $\boldsymbol{b}_{k_{1} \ldots k_{n}}$, with $k_{1}+\cdots+k_{n}=1$. According to the adopted expansion basis, the nonlinear maps discussed above are referred as JT-based $k$ th-order Taylor nonlinear map method (JTTNP-k), and JT-based $k$ th-order Chebyshev nonlinear map method (JTCNP-k).

An important issue that has to be pointed out here is related with the computational efficiency. Note that the computational time when using JTTNP-k or JTCNP-k expansions includes both the time required for computing the polynomial formulae (this is, $\mathcal{T}^{k}, C^{k}$ ) and the time needed for evaluating at all the required values, using $\mathcal{T}^{k}$ or $C^{k}$. It is clear that the computational time for the first task is much longer than its numerical evaluation at a single point. In 
contrast, once the polynomial results are computed, the computational time for the repeated evaluations using $\mathcal{T}^{k}$ or $C^{k}$ is much faster than the common pointwise Monte Carlo numerical simulation. In particular, for a given nonlinear map, the computations of the first part are independent on the number of sampling points or initial conditions to be used, but for the second part, the time devoted to the evaluation of a polynomial is much less than a full pointwise numerical Monte Carlo simulation. Therefore, as the number of sampling points, or initial conditions, increases, the computational efficiency of JTTNP-k or JTCNP-k also does. The computational effort for computing $\mathcal{T}^{k}$ or $C^{k}$ mainly depends on the expansion order, the complexity of the function, the choice of Taylor or Chebyshev series, as well as the integration scheme and accuracy requirement when an ODE system is involved in the nonlinear map evaluation. In [16] the authors showed that a $k$ th-order Taylor expansion map is more efficient than the classical nonlinear pointwise Monte Carlo method in general circumstances. This fact is assumed and will not be validated in this work; our main focus will be on the comparison of the computational efficiency of JTTNP-k expansions with respect to JTCNP-k expansions.

Another interesting point is that the JT procedure can also include parameter uncertainties $\delta \boldsymbol{p}_{0}$ in the final result. This can be seen just assuming that some of the $\boldsymbol{x}$ variables are parameters, but it can be made more explicit according to what follows. Consider, for instance, an ODE system $\dot{\boldsymbol{x}}=\boldsymbol{f}(\boldsymbol{x}, \boldsymbol{p}, t)$ with a $n$-dimensional initial state vector $\boldsymbol{x}\left(t_{0}\right)=\boldsymbol{x}_{0}$, and a $l$-dimensional initial parameter vector $\boldsymbol{p}\left(t_{0}\right)=\boldsymbol{p}_{0}$. Let the associated solution flow be denoted by $\boldsymbol{x}(t)=\boldsymbol{\Phi}\left(t ; t_{0}, \boldsymbol{x}_{0}, \boldsymbol{p}_{0}\right)$. The JT method propagates a neighborhood $\mathcal{N}$ around $\boldsymbol{x}_{0}$ and $\boldsymbol{p}_{0}$ from $t_{0}$ up to a final time $t_{d}$. In the JTTNP-k case, the neighborhood $\mathcal{N}$ is parameterized by a set of variables $\left[\boldsymbol{x}_{0}\right]_{T}=\overline{\boldsymbol{x}}_{0}+\delta \boldsymbol{x}_{\mathbf{0}}$ and $\left[\boldsymbol{p}_{0}\right]_{T}=\overline{\boldsymbol{p}}_{0}+\delta \boldsymbol{p}_{\mathbf{0}}$. After the integration along the time interval $\left[t_{0}, t_{1}\right]$, the resulting Taylor expansion of the solution flow $\boldsymbol{\Phi}\left(t_{0}+h ; t_{0}, \boldsymbol{x}_{0}+\boldsymbol{\delta} \boldsymbol{x}_{\mathbf{0}}, \boldsymbol{p}_{0}+\delta \boldsymbol{p}_{\mathbf{0}}\right)$ is expressed as $\left[\boldsymbol{x}_{1}\right]_{T}^{k}=\overline{\boldsymbol{x}}_{1}+\mathcal{T}_{\boldsymbol{x}_{1}}^{k}\left(\delta \boldsymbol{x}_{0}, \delta \boldsymbol{p}_{\mathbf{0}}\right)$. This expansion is the one used as new initial value in the next numerical integration step $\left[t_{1}, t_{2}\right]$. The procedure will be repeated until a final epoch $t_{d}$ be reached, providing the desired $k$ th-order Taylor expansion of the solution flow, that is,

$$
\begin{aligned}
{\left[\boldsymbol{x}_{d}\right]_{T}^{k} } & =\overline{\boldsymbol{x}}_{d}+\mathcal{T}_{\boldsymbol{x}_{d}}^{k}\left(\delta \boldsymbol{x}_{0}, \delta \boldsymbol{p}_{0}\right) \\
& =\sum_{0 \leq k_{1}+\cdots+k_{n+l} \leq k} \boldsymbol{a}_{k_{1} \ldots k_{n+l}} \delta x_{0,1}^{k_{1}} \ldots \delta x_{0, n}^{k_{n}} \delta p_{0,1}^{k_{n+1}} \ldots \delta p_{0, l}^{k_{n+l}},
\end{aligned}
$$

where $\delta x_{0,1}, \ldots, \delta x_{0, n}$ denote the components of the initial state deviation $\delta \boldsymbol{x}_{0} ; \delta p_{0,1}, \ldots, \delta p_{0, l}$ are the components of the initial parameter deviation $\delta \boldsymbol{p}_{0}$, while $\boldsymbol{a}_{k_{1} \ldots k_{n+l}}$ represent the coefficients of the Taylor expansion of the solution flow.

In the JTCNP-k case, the neighborhood should be first mapped into the interval $[-1,1]$ and it is apparent that the size of the neighborhood affects the accuracy when the order of the Chebyshev expansion is fixed. However, analogously 
to the Taylor procedure, we calculate the $k$ th-order Chebyshev expansion of the solution flow:

$$
\begin{aligned}
{\left[\boldsymbol{x}_{d}\right]_{C}^{k} } & =\overline{\boldsymbol{x}}_{d}+C_{\boldsymbol{x}_{d}}^{k}\left(\delta \boldsymbol{x}_{0}, \delta \boldsymbol{p}_{0}\right) \\
& =\sum_{0 \leq k_{1}+\cdots+k_{n+l} \leq k} \boldsymbol{b}_{k_{1} \ldots k_{n+l}} T_{k_{1}}\left(\delta x_{0,1}\right) \ldots T_{k_{n}}\left(\delta x_{0, n}\right) T_{k_{n+1}}\left(\delta p_{0,1}\right), \ldots T_{k_{n+l}}\left(\delta p_{0, n+l}\right),
\end{aligned}
$$

where now $\boldsymbol{b}_{k_{1} \ldots k_{n+l}}$ represent the coefficients of Chebyshev expansion of the solution flow.

\section{JT-based Runge Kutta integrator}

In order to perform a JT-based numerical integration, all the arithmetic operations of the usual numerical propagator must be replaced with the corresponding polynomial algebra operations in the JT scheme. After each time step, the state $\boldsymbol{x}$ and the right hand side of the ODE system $\dot{\boldsymbol{x}}=\boldsymbol{f}(\boldsymbol{x}, t)$ is expressed as a $k$ th-order expansion around a certain point of the nominal solution. At this point, it is worth to remark a particularity of the JT-based numerical integrators with adaptive step size control, such as the Runge-Kutta-Fehlberg procedures.

The Runge-Kutta (RK) methods are the only ones we consider in our study. These methods can be divided in two categories, including fixed or variable stepsize. In general, a RK with variable stepsize, such as the well known RK45 and RK78 of respective orders 5 and 8, gives a better performance but, some care has to be taken in the stepsize control when doing JT propagations. In the following, some remarks about the implementation of a JTRK78 algorithm are discussed in detail; other implementations, like the one of JTRK45, can be performed using the similar error estimation and stepsize control strategy.

Consider the initial value problem in a vectorfield with $n$ variables. Assume a well approximated JT-state $\left[\boldsymbol{x}_{j}\right]_{*}^{k}$ at

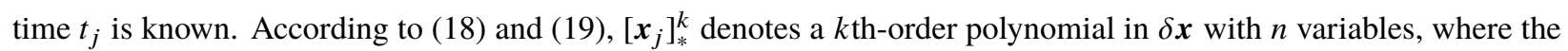
subscript $*$ indicates either Taylor or Chebyshev expansions. For a given time step $h$, two estimates of the real state $\boldsymbol{x}\left(t_{j+1}\right)$ at $t_{j+1}=t_{j}+h$, are provided by the seventh and eighth order JTRK, respectively:

$$
\left[\overline{\boldsymbol{x}}_{j+1}\right]_{*}^{k}=\left[\boldsymbol{x}_{j}\right]_{*}^{k}+h \boldsymbol{\phi}_{7}\left(t_{j},\left[\boldsymbol{x}_{j}\right]_{*}^{k}, h\right), \quad\left[\hat{\boldsymbol{x}}_{j+1}\right]_{*}^{k}=\left[\boldsymbol{x}_{j}\right]_{*}^{k}+h \boldsymbol{\phi}_{8}\left(t_{j},\left[\boldsymbol{x}_{j}\right]_{*}^{k}, h\right) .
$$

The fundamental idea underlying a RK method with variable stepsize control is that when the two estimations are close enough (this is, a suitable norm of their difference is below a selected threshold value $\epsilon$ ), the propagation with stepsize $h$ is considered successful, while when they differ, the stepsize must be recomputed and adjusted. A trade-off between a fixed accuracy and the computational time, requires also to look for the maximum stepsize which could be suitable to obtain a good estimation the real state $\boldsymbol{x}\left(t_{j+1}\right)$ when the estimators differ, as well as for the computation of $\boldsymbol{x}\left(t_{j+2}\right)$ at time $t_{j+2}$ that will follow in the propagation. 
Note that, at time step $j$, the difference between the two estimations is an expansion of the form,

$$
\left[\overline{\boldsymbol{x}}_{j+1}^{i}\right]_{*}^{k}-\left[\hat{\boldsymbol{x}}_{j+1}^{i}\right]_{*}^{k}=\sum_{0 \leq m \leq k} \boldsymbol{c}_{m}^{j} \boldsymbol{\delta} \boldsymbol{x}_{m}=\sum_{0 \leq k_{1}+\cdots+k_{n} \leq k} \boldsymbol{c}_{k_{1} \ldots k_{n}}^{j} \delta x_{1}^{k_{1}} \ldots \delta x_{n}^{k_{n}}
$$

The classical pointwise propagation algorithms estimate this difference considering some norm of the $n$-vector $\boldsymbol{c}_{0 . \ldots 0}^{j}$, but in our case (23]) is a $k$ th-order expansion in $n$ variables and all terms must be taken into account. Assuming a threshold for each order, $\epsilon=\left(\epsilon_{1} \ldots \epsilon_{k}\right)$ we can define a norm by ${ }^{\text {* }:}$

$$
C_{m}^{j}=\max _{k_{1}+\ldots+k_{n}=m}\left\|\boldsymbol{c}_{k_{1} \ldots k_{n}}^{j}\right\|, \quad\left\|\boldsymbol{c}_{k_{1} \ldots k_{n}}^{j}\right\|=\max _{1 \leq i \leq n}\left|c_{i, k_{1} \ldots k_{n}}^{j}\right|
$$

The accuracy condition for a successful time step $h$ at time $t_{j}$ would be $C_{m}^{j}<\epsilon_{m}$ for $m=0 \ldots k$. In order to further determine the optimal stepsize for a given accuracy $\boldsymbol{\epsilon}$, let us first introduce:

$$
\boldsymbol{\Delta}(t, \boldsymbol{x}, h)=\frac{\boldsymbol{x}(t+h)-\boldsymbol{x}(t)}{h}
$$

Since the JTRK7 has a 7th-order local truncation error, we can write:

$$
-\Delta\left(t_{j}, \boldsymbol{x}_{j}, h\right)+\boldsymbol{\phi}_{7}\left(t_{j},\left[\boldsymbol{x}_{j}\right]_{*}^{k}, h\right)=N_{7}\left(t_{j}\right) h^{7}+O\left(h^{8}\right) .
$$

Analogously, JTRK8 has 8th order accuracy and thus:

$$
-\boldsymbol{\Delta}\left(t_{j}, \boldsymbol{x}_{j}, h\right)+\boldsymbol{\phi}_{8}\left(t_{j},\left[\boldsymbol{x}_{j}\right]_{*}^{k}, h\right)=\boldsymbol{N}_{8}\left(t_{j}\right) h^{8}+O\left(h^{9}\right) .
$$

Subtracting the estimations given by (22), and using ([26-[27), we get:

$$
\left[\overline{\boldsymbol{x}}_{j+1}\right]_{*}^{k}-\left[\hat{\boldsymbol{x}}_{j+1}\right]_{*}^{k}=h\left(\boldsymbol{\phi}_{7}-\boldsymbol{\phi}_{8}\right)=\boldsymbol{N}_{7}\left(t_{j}\right) h^{8}+O\left(h^{9}\right) .
$$

Neglecting the $O\left(h^{9}\right)$ term we obtain the following estimate of the leading coefficient in the truncation:

$$
N_{7}\left(t_{j}\right) \simeq \frac{\left[\bar{x}_{j+1}\right]_{*}^{k}-\left[\hat{x}_{j+1}\right]_{*}^{k}}{h^{8}}
$$

and, according to (24), we have the following estimation for the size of the different orders of $N_{7}\left(t_{j}\right)$ :

$$
N_{7, m}^{j}=\frac{C_{m}^{j}}{h^{8}}, \quad m=0, \ldots, k .
$$

\footnotetext{
*Other definitions of $C_{m}^{j}$ like scaled Euclidean norms or average of absolute values among components could be also considered.
} 
Let us now assume a new optimal time step $h_{N}$ at time $t_{j+1}$. According to (BDi) together with the successful condition, we get:

$$
C_{m}^{j+1}=h_{N}^{8} N_{7, m}^{j+1} \leq \epsilon_{m}, \quad m=0, \ldots, k .
$$

Expanding $\boldsymbol{N}_{7}\left(t_{j+1}\right)$ around $t_{j}$ yields $\boldsymbol{N}_{7}\left(t_{j+1}\right)=\boldsymbol{N}_{7}\left(t_{j}+h_{N}\right)=\boldsymbol{N}_{7}\left(t_{j}\right)+O\left(h_{N}\right)$, and so (BII) can be written as:

$$
C_{m}^{j+1}=h_{N}^{8} N_{7, m}^{j}+O\left(h_{N}^{9}\right) \leq \epsilon_{m}, \quad m=0, \ldots, k .
$$

Then, neglecting the $O\left(h_{N}^{9}\right)$ term, and substituting (BO) into (32), we obtain:

$$
h_{N, m} \leq \sqrt[8]{\frac{\epsilon_{m}}{N_{7, m}^{j}}}=h \sqrt[8]{\frac{\epsilon_{m}}{C_{m}^{j}}}, \quad m=0, \ldots, k
$$

The value of $h_{N}$ advised for the next step propagation is the minimum of these $k$ values. Usually $h_{N}$ is multiplied by a safety factor of 0.9 (in practice, this avoids re-computations of the stepsize and increases performance).

\section{Geostationary trajectory propagation}

During the propagation of geotrajectory uncertainties, some certain nonlinear transformations, including the conversion from GEO elements to Cartesian coordinates and the impact of position deviation on the perturbing accelerations, are frequently repeated. The selection of the expansion order for these nonlinear maps not only affects the accuracy, but also plays a significant role on the total computational effort. A detailed analysis is useful to account for the optimal expansion order that keeps the required accuracy at the least cost; it also allows to determine the comparative performance of JTTNP-k and JTCNP-k.

\section{Statistical definitions}

As it has already been said, the JTTNP-k and JTCNP-k methods enable to obtain polynomials $\mathcal{T}_{\boldsymbol{x}}^{k}\left(\delta \boldsymbol{x}_{0}, \delta \boldsymbol{p}_{0}\right)$ and $C_{\boldsymbol{x}}^{k}\left(\delta \boldsymbol{x}_{0}, \delta \boldsymbol{p}_{0}\right)$, for accurately mapping initial deviations $\delta \boldsymbol{x}_{0}$ and $\delta \boldsymbol{p}_{0}$ into a final value or state $\boldsymbol{x}$. We are going to assess the performance of both methods by comparing their accuracy, computational time, and error distribution over the whole uncertainty neighborhood. The error is defined as the difference at $N$ points between the results, $\boldsymbol{x}_{j}^{k}$, computed using either JTTNP-k or JTCNP-k, and the reference value, $\widetilde{\boldsymbol{x}}_{j}$, computed by means of an accurate numerical pointwise evaluation at the same sampling data points. This is, $\boldsymbol{\varepsilon}_{j}^{k}=\widetilde{\boldsymbol{x}}_{j}-\boldsymbol{x}_{j}^{k}, j=1, \ldots, N$. The average errors in the components are defined as:

$$
\varepsilon_{\mathrm{ave}}^{k, i}=\frac{1}{N} \sum_{j=1}^{N}\left|\tilde{\boldsymbol{x}}_{j}^{i}-\boldsymbol{x}_{j}^{k, i}\right|,
$$


where $i$ indicates the $i$ th state variable. In principle, either the JTTNP-k or the JTCNP-k expansions can be used for the applications, as far as the error of any arbitrary state variable $\boldsymbol{x}^{k, i}$ satisfies the condition $\boldsymbol{\varepsilon}_{\text {ave }}^{k, i} \leq \boldsymbol{\varepsilon}_{\max }^{i}$, where $\boldsymbol{\varepsilon}_{\max }$ is an error threshold vector.

Before using JTTNP-k and JTCNP-k expansions for the study of the propagations of geotrajectory uncertainties, we also focus on their behaviors for some nonlinear mappings implemented, including the transformation from the GEO elements to Cartesian coordinates and the map from position deviation to perturbing accelerations required in the GEO trajectory propagation. In these procedures we define the average position and velocity errors as:

$$
\varepsilon_{\mathrm{ave}, \mathrm{r}}^{k}=\frac{1}{N} \sum_{j=1}^{N} \sqrt{\left(\varepsilon_{j}^{k, x}\right)^{2}+\left(\varepsilon_{j}^{k, y}\right)^{2}+\left(\varepsilon_{j}^{k, z}\right)^{2}}, \quad \varepsilon_{\mathrm{ave}, \mathrm{v}}^{k}=\frac{1}{N} \sum_{j=1}^{N} \sqrt{\left(\varepsilon_{j}^{k, \dot{x}}\right)^{2}+\left(\varepsilon_{j}^{k, \dot{y}}\right)^{2}+\left(\varepsilon_{j}^{k, \dot{z}}\right)^{2}},
$$

where the $i$ th component in $\boldsymbol{\varepsilon}_{j}^{k, i}=\widetilde{\boldsymbol{x}}_{j}^{i}-\boldsymbol{x}_{j}^{k, i}$ is now denoted by, $i=(x, y, z, \dot{x}, \dot{y}, \dot{z})$.

To assess the resulting error distribution of JTTNP-k and JTCNP-k expansions, the variances of position and velocity errors will be also considered:

$$
\sigma_{\mathrm{r}}^{k}=\sqrt{\sigma_{x}^{2}+\sigma_{y}^{2}+\sigma_{z}^{2}}, \quad \sigma_{\mathrm{V}}^{k}=\sqrt{\sigma_{\dot{x}}^{2}+\sigma_{\dot{y}}^{2}+\sigma_{\dot{z}}^{2}}
$$

where

$$
\sigma_{i}=\frac{1}{N-1} \sqrt{\sum_{j=1}^{N}\left(\varepsilon_{j}^{k, i}-\varepsilon_{\mathrm{ave}}^{k, i}\right)^{2}}, \quad i=(x, y, z, \dot{x}, \dot{y}, \dot{z})
$$

Similarly, we define the average perturbing acceleration error to assess the performance of JTTNP-k and JTCNP-k expansions for mapping the position deviation to the selected perturbing acceleration, as:

$$
\varepsilon_{\mathrm{a}, \mathrm{ave}}^{k}=\frac{1}{N} \cdot \sum_{j=1}^{N} \sqrt{\left(\varepsilon_{j}^{k, \ddot{x}}\right)^{2}+\left(\varepsilon_{j}^{k, \ddot{y}}\right)^{2}+\left(\varepsilon_{j}^{k, \ddot{z}}\right)^{2}}
$$

where $\left(\varepsilon_{j}^{k, \ddot{x}}, \varepsilon_{j}^{k, \ddot{y}}, \varepsilon_{j}^{k, \ddot{z}}\right)$ stands for the acceleration error vector in the samples.

Finally, let us briefly comment some results on the behavior of the probability density function (PDF) of a propagated uncertainty neighborhood. Using the $k$ th-order expansion of a flow map, if the initial state and parameter PDFs are known (i.e. $p\left(\mathbf{x}_{0}\right)$ and $\left.p\left(\mathbf{p}_{0}\right)\right)$, the final state PDF, $p(\boldsymbol{x})$, as well as its main cumulants, can be computed in the JT framework. For a random variable $\boldsymbol{x}$ related to $\boldsymbol{x}_{0}$ and $\boldsymbol{p}_{0}$, the components of first two moments can be computed as:

$$
\begin{aligned}
\boldsymbol{m}_{i} & =E\left\{\left[x_{i}\right]\right\}, \\
\boldsymbol{P}_{i j} & =E\left\{\left(\left[x_{i}\right]-m_{i}\right)\left(\left[x_{j}\right]-m_{j}\right)\right\},
\end{aligned}
$$

where $\boldsymbol{m}$ and $\boldsymbol{P}$ denote the mean and the covariance of the transformed PDF corresponding to the propagation of the 
trajectory uncertainties. Therefore, the first two moments can be evaluated by substituting (201) into (B6)). To illustrate this process, the first two moments are computed using Taylor series as follows:

$$
\begin{aligned}
\boldsymbol{m}_{i}^{k} & =\sum_{0 \leq k_{1}+\cdots+k_{n+l} \leq k} \boldsymbol{a}_{i, k_{1} \ldots k_{n+l}} E\left\{\delta x_{1}^{k_{1}} \ldots \delta x_{n}^{k_{n}} \delta p_{0,1}^{k_{n+1}} \ldots \delta p_{0, l}^{k_{n+l}}\right\}, \\
\boldsymbol{P}_{i j}^{k} & =\sum_{\substack{0 \leq k_{1}+\cdots+k_{n+l} \leq k, 0 \leq q_{1}+\cdots+q_{n+l} \leq k}} \boldsymbol{c}_{i, k_{1} \ldots k_{n+l}} \boldsymbol{c}_{j, q_{1} \ldots q_{n+l}} E\left\{\delta x_{1}^{k_{1}+q_{1}} \ldots \delta x_{n}^{k_{n}+q_{n}} \delta p_{0,1}^{k_{n+1}+q_{n+1}} \ldots \delta p_{0, l}^{k_{n+l}+q_{n+l}}\right\},
\end{aligned}
$$

where the calculation of $\boldsymbol{c}_{i, k_{1} \ldots k_{n+l}}, \boldsymbol{c}_{j, q_{1} \ldots q_{n+l}}$ in (B8) is done incorporating $\boldsymbol{a}_{i, k_{1} \ldots k_{n+l}}$ in (188) with the subtraction of their mean value terms, this is, $\boldsymbol{c}_{i, 0}=\boldsymbol{a}_{i, 0}-\boldsymbol{m}_{i}^{k}$, and $\boldsymbol{c}_{i, k_{1} \ldots k_{n+l}}=\boldsymbol{a}_{i, k_{1} \ldots k_{n+l}}$ when $k>0$. It is clear that the effort for computing the expectation increases with the expansion order, the number of states and parameters in the model, as well as the order of the estimated moments.

An analogous numerical procedure can be implemented to estimate the final state PDF for the Chebyshev case by means of converting the Chebyshev basis into the monomial basis. We note that in this case the size of uncertainty neighborhood should be predetermined, and all the state and parameter deviations should be involved in the uncertainty neighborhood. Again, this is different with respect to the Taylor case, in which is not necessary to consider the size of the uncertainty neighborhood a priori.

We note that for these nonlinear propagations, no hypothesis is made on the initial state PDF, $\boldsymbol{p}\left(\boldsymbol{x}_{0}\right)$, and the parameter PDF, $\boldsymbol{p}\left(\boldsymbol{p}_{0}\right)$. This is, any arbitrary initial random variable distribution can be mapped by the JTTNM-k and JTCNM-k methods. In many applications, an initial Gaussian random variable (GRV) is frequently assumed due to its nice statistical properties, and it can be completely described by means of the first two moments. Furthermore, if the mean of the GRV distribution is zero, Isserliss formula [23] provides an analytical expression to compute the resulting expectation values in (37, [88). Thus, the higher-order moments of the transformed PDF are evaluated by means of (36).

\section{Transforming GEO elements into Cartesian coordinates}

Since the perturbing acceleration is easily modeled in Cartesian coordinates, it is necessary to evaluate the spacecraft Cartesian coordinates in terms of the GEO elements during the propagations of the GEO model. At a given epoch $t$, (III) explicitly relates the GEO elements to the Cartesian coordinates, this is, $\boldsymbol{x}_{c}=\boldsymbol{g}\left(\boldsymbol{x}_{G}\right)$.

Using the JTTNP-k and JTCNP-k expansions, discussed in Section II, we define the GEO elements as JT variables according to,

$$
\left[\boldsymbol{x}_{G}\right]=\overline{\boldsymbol{x}}_{G}+\delta \boldsymbol{x}_{G}
$$

Following ([18) or (19), we perform the nonlinear conversion from GEO elements to Cartesian coordinates, either 
by means of Taylor or Chebyshev series, as follows:

$$
\begin{aligned}
& {\left[\boldsymbol{x}_{c}\right]_{T}^{k}=\overline{\boldsymbol{x}}_{c}+\mathcal{T}_{\boldsymbol{x}_{c}}^{k}\left(\delta \boldsymbol{x}_{G}\right)=\sum_{0 \leq k_{1}+\cdots+k_{6} \leq k} \boldsymbol{a}_{k_{1} \ldots k_{6}} \delta x_{G, 1}^{k_{1}} \ldots \delta x_{G, 6}^{k_{6}},} \\
& {\left[\boldsymbol{x}_{c}\right]_{C}^{k}=\overline{\boldsymbol{x}}_{c}+C_{\boldsymbol{x}_{c}}^{k}\left(\delta \boldsymbol{x}_{G}\right)=\sum_{0 \leq k_{1}+\cdots+k_{6} \leq k} \boldsymbol{b}_{k_{1} \ldots k_{6}} T_{k_{1}}\left(\delta x_{G, 1}\right) \ldots T_{k_{6}}\left(\delta x_{G, 6}\right) .}
\end{aligned}
$$

These two polynomial expansions enable to map the deviations of the GEO elements to Cartesian coordinates in a nonlinear and efficient way. In other words, they describe the nonlinear dependence of Cartesian coordinates on the changes of the GEO elements. Table $\square$ gives the nominal GEO elements and their associated uncertainty neighborhoods (denoted as $\Delta$ ) used for the simulations.

Table 1 Nominal GEO elements, associated uncertainty neighborhoods, as well as spacecraft paramters

\begin{tabular}{ccc}
\hline Parameter & Nominal value & Uncertainty neighborhood $\Delta$ \\
\hline Epoch & 15 November 2015, 0:0:0 UTC & - \\
$\lambda$ & $5.34479641266 \mathrm{rad}$ & $10^{-3} \mathrm{rad}$ \\
$\delta \bar{a}$ & $-1.42088440198 \times 10^{-6}$ & $10^{-3}$ \\
$e_{x}$ & 0 & $10^{-3}$ \\
$e_{y}$ & 0 & $10^{-3}$ \\
$Q_{1}$ & 0 & $10^{-3}$ \\
$Q_{2}$ & 0 & $10^{-3}$ \\
illuminated area & $74 \mathrm{~m}^{2}$ & - \\
mass & $3300 \mathrm{~kg}$ & - \\
$C_{r}$ & 1.3 & - \\
\hline
\end{tabular}

To save computational time, the lowest expansion order for coordinate conversion, using both JTTNP-k and JTCNP-k methods, and satisfying the error requirements, is advisable. This lowest order will be referred as the optimal expansion order. For its determination we consider a multivariable uniform distribution over the uncertainty neighborhood of the GEO elements, that is: $\left[x_{G}-\Delta, x_{G}+\Delta\right]$.

Figure $\mathbb{W}$ shows the average position and velocity errors (B3]) of the coordinate conversion, using JTTNP-k and JTCNP-k. From the figure it can be concluded that both average errors are of the same order of magnitude, and decrease exponentially with the expansion order. In particular, when the expansion order exceeds four, the practical convergence is not improved due to the floating point representation accuracy.

Table $\square$ gives the position and the velocity error variances (B4) of the coordinate transformation. Note that, for a fixed expansion order, the error variance of JTCNP-k is smaller than the one of JTTNP-k, meaning that the error distribution of JTCNP-k behaves in a more uniform way. To better explain this fact, consider only an uncertainty in the $\lambda$ element. Figure $\rrbracket$ shows the position and velocity errors using JTTNP-3 and JTCNP-3. From this figure, it is clear that JTCNP-3 possesses an uniform error distribution over the interval $[\lambda-\Delta \lambda, \lambda+\Delta \lambda]$, while JTTNP-3 
has an extremely good accuracy around the expansion point, as is usual in Taylor expansions, but the errors increase when moving away from it. Similar conclusions are obtained when the expansion order is less than four. If the expansion order exceeds four, both JTTNP-k and JTCNP-k methods are accurate enough to approximate the nonlinear coordinate transformation and the error distribution is almost the same albeit, of course, of the limits of the floating point representation accuracy. The fact that, for a fixed expansion order $k$, the JTCNP-k error is much smaller than the one of JTTNP-k at the edge of the uncertainty neighborhood, reveals that JTCNP-k can map a bigger uncertainty neighborhood for the same expansion order and accuracy requirements. For instance, when the accuracy threshold in position is $4 \times 10^{-7} \mathrm{~m}$, JTTNP-3 is effective over the uncertainty interval [ $\left.-0.0007,0.0007\right]$, while JTCNP-3 is effective over the whole uncertainty interval $[-0.001,0.001]$. Similar conclusions hold enlarging the uncertainty neighborhood to $[-0.01,0.01]$, as shown in Fig. [1].
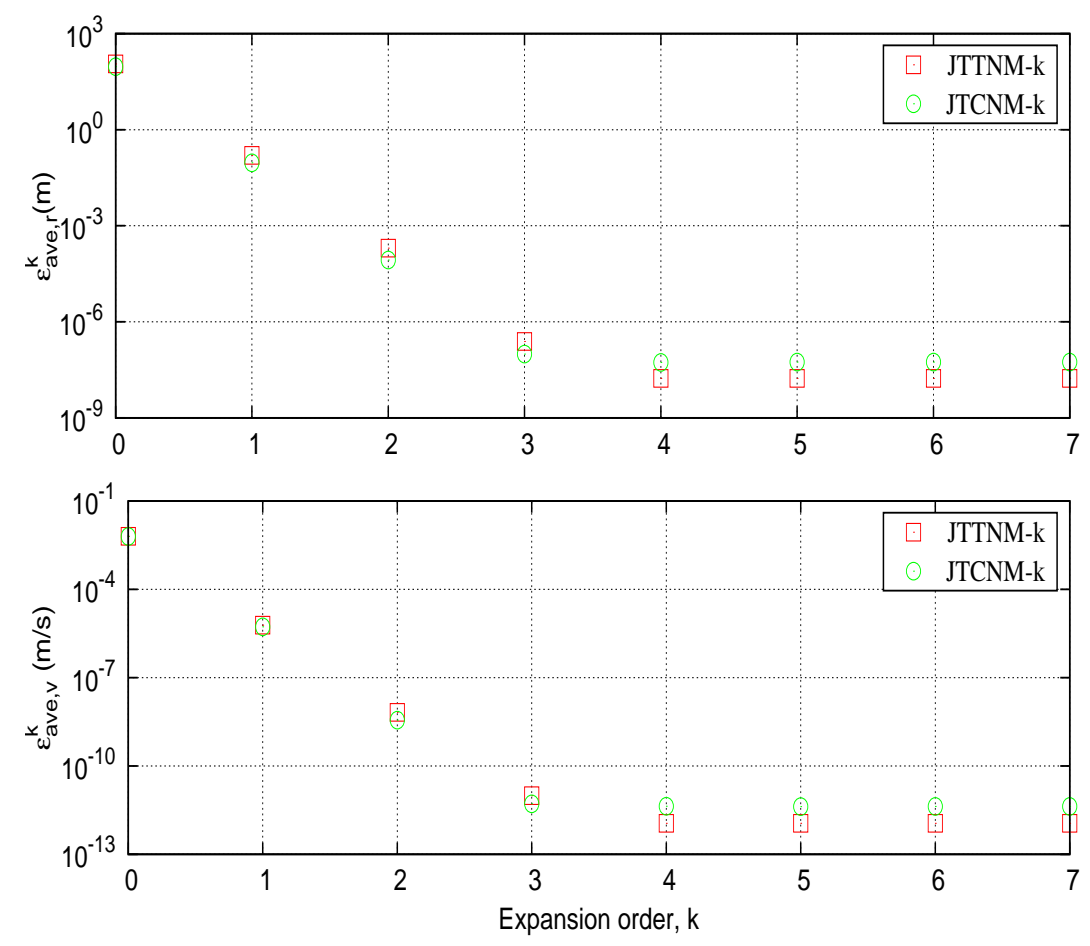

Fig. 1 Accuracy of JTTNP-k and JTCNP-k expansions for the transformation from GEO elements to Cartesian coordinates.

Figure 4 shows the computational time required by both JTTNP-k and JTCNP-k methods for the coordinate transformation. Clearly, the computational effort associated to these methods increases with the expansion order, and the JTTNP-k procedure is several orders of magnitude more efficient than the JTCNP-k. This phenomenon becomes more prominent when higher order expansion are used, meaning that JTCNP-k would be difficult to be considered for these cases. The above discussion shows that, although the average errors of the JTTNP-k and JTCNP-k methods are on the same order of magnitude, some other considerations must be done: from one side, the computational effort of the 
Table 2 Error variance for the transformation from GEO elements to Cartesian coordinates. Beyond order 5 no further improvement is observed due to numerical accuracy.

\begin{tabular}{ccccc}
\hline \multirow{2}{*}{ Expansion order } & \multicolumn{3}{c}{ Position error variance $\left(\mathrm{m}^{2}\right)$} & \multicolumn{2}{c}{ Velocity error variance $\left((\mathrm{m} / \mathrm{s})^{2}\right)$} \\
& JTTNP-k & JTCNP-k & JTTNP-k & JTCNP-k \\
\hline 1 & 2886.2 & 1871.0 & $1.3 \times 10^{-5}$ & $1.1 \times 10^{-5}$ \\
2 & $1 \times 10^{-2}$ & $3 \times 10^{-3}$ & $1.9 \times 10^{-11}$ & $1.1 \times 10^{-11}$ \\
3 & $3 \times 10^{-8}$ & $5 \times 10^{-9}$ & $3.4 \times 10^{-17}$ & $8.2 \times 10^{-18}$ \\
4 & $6 \times 10^{-14}$ & $5 \times 10^{-15}$ & $8.1 \times 10^{-23}$ & $6.2 \times 10^{-24}$ \\
5 & $6 \times 10^{-14}$ & $8 \times 10^{-16}$ & $4.9 \times 10^{-25}$ & $2.0 \times 10^{-25}$ \\
\hline
\end{tabular}
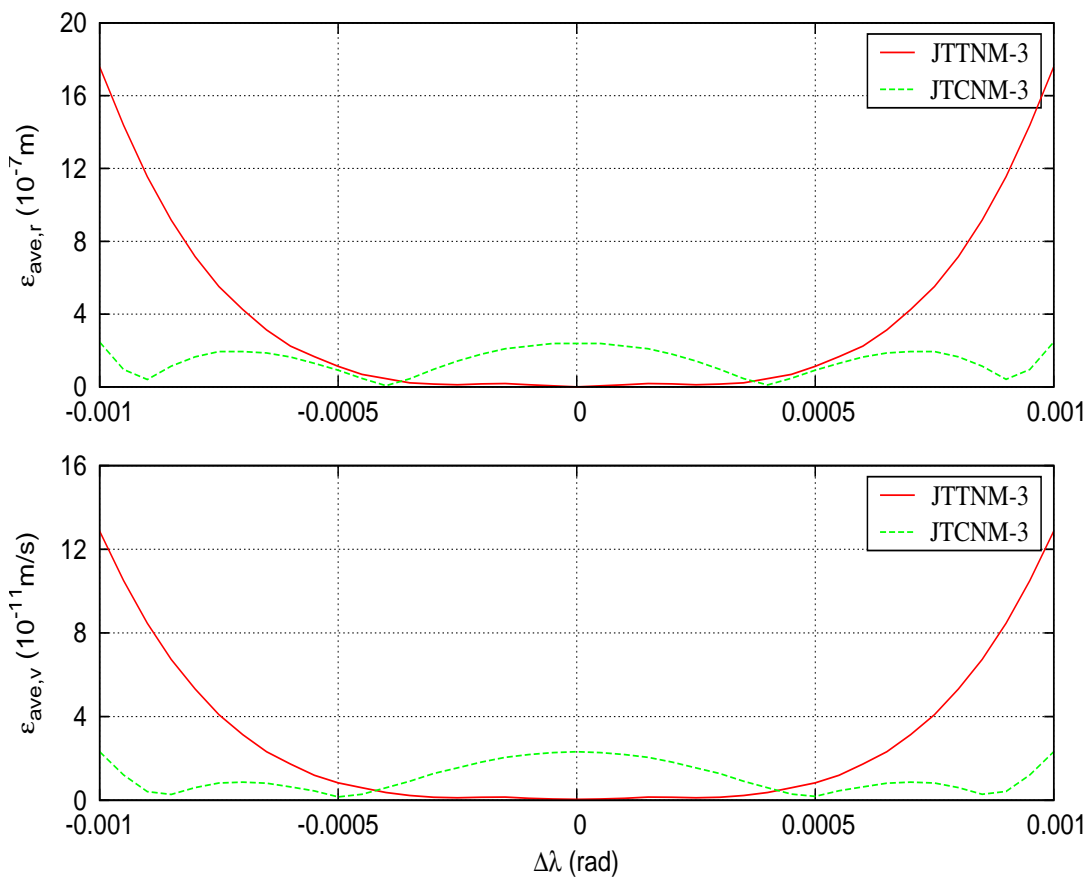

Fig. 2 Accuracy of JTTNP-3 and JTCNP-3 expansions for the transformation from GEO elements to Cartesian, accounting only for the uncertainty $[-0.001,0.001]$ on the element $\lambda$. 

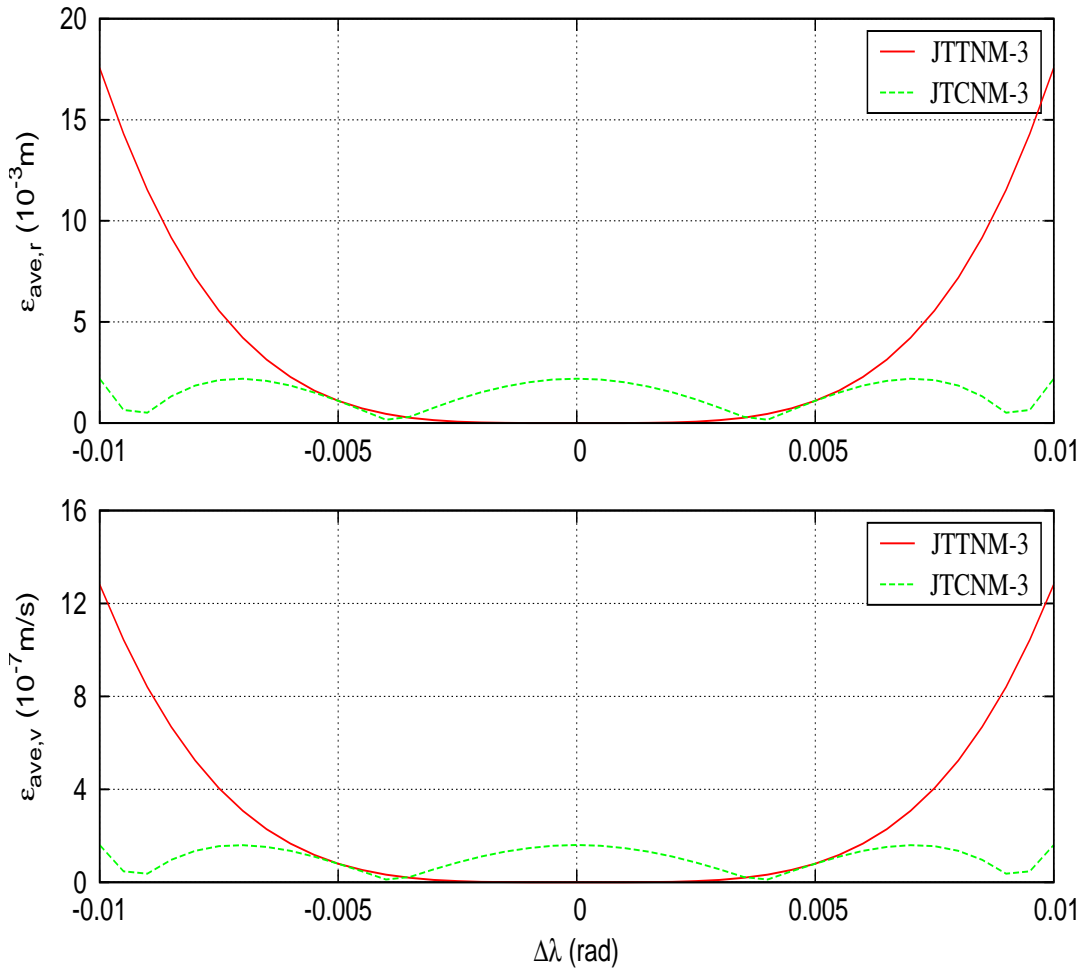

Fig. 3 Accuracy of JTTNP-3 and JTCNP-3 expansions for the transformation from GEO elements to Cartesian, accounting only for the uncertainty $[-0.01,0.01]$ on the element $\lambda$.

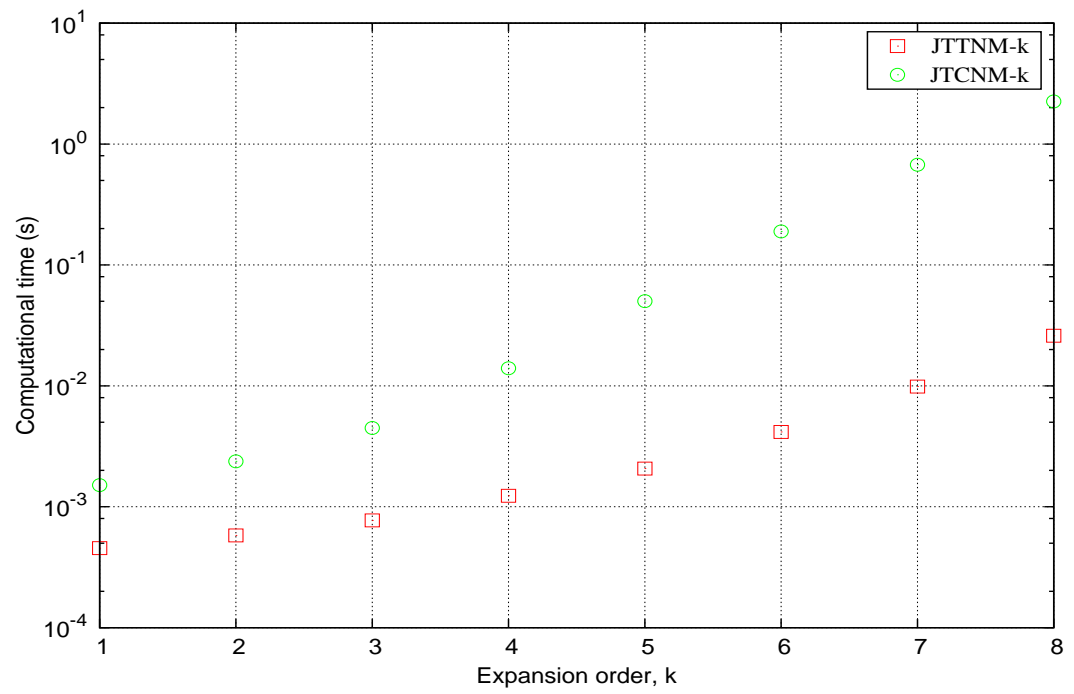

Fig. 4 Computational time associated to JTTNP-k and JTCNP-k in the transformation from GEO elements to Cartesian coordinates (implemented on an Intel(R) Core(TM) i5-7300HQ under GNU-Linux C++ compiler). 
JTTNP-k method has a better behavior, however, JTCNP-k possesses a smoother error distribution over the uncertainty neighborhood and a smaller error at its edges. Therefore, for a prescribed accuracy threshold, JTCNP-k method allows to map a bigger uncertainty neighborhood. Of course, since the computational speed of the JTTNP-k method is faster, we could split the uncertainty neighborhood and apply the JTTNP-k method on each sub-neighborhood [18, 19]. Based on this idea, a deeper investigation about the trade-off between the JTTNP-k and JTCNP-k methods should be done, but the complexity of the number of cases to be analyzed exceeds the needs and purpose of the present paper.

\section{Perturbing accelerations computation}

During an orbit propagation using the Jet transport method, the vectorfield, which includes all the orbital perturbations, needs to be evaluated. Consequently, most of the computational cost of the propagation is associated to the evaluation of the orbital perturbations and, as a consequence, a trade-off between accuracy and computational time must be done for the application of the JTTNP-k and JTCNP-k methods (18-19). The goal of this section is to explore the relations between the expansion order, the accuracy requirements, and the size of the uncertainty neighborhood in the computation of the perturbing accelerations.

For the perturbing accelerations acting on a geostationary spacecraft we have set the accuracy threshold equal to $10^{-11} \mathrm{~m} / \mathrm{s}^{2}$. According to [24], this value is three orders of magnitude smaller than the solar radiation pressure acceleration, which is the smallest one among the four dominant perturbations acting on a GEO spacecraft; thus, the influence of errors smaller than $10^{-11} \mathrm{~m} / \mathrm{s}^{2}$ are practically negligible. Using this accuracy threshold, we aim to study the lowest expansion order required for different sizes of the uncertainty neighborhood. This lowest order will be the optimal expansion order for each perturbing acceleration.

The accuracy analysis of the JTTNP-k and JTCNP-k methods for each perturbation is performed as follows. We consider a particular position of the GEO spacecraft (for the geopotential perturbation this particular position depends on the sub-satellite longitude, for the SRP or Sun and Moon third-body perturbations, it depends on the relative positions of these bodies) and around it we consider a multivariable uniform distribution over an uncertainty neighborhood where we sample. Next, we perform the nonlinear transformation for mapping the current GEO position uncertainty into suitable coordinates in order to estimate the acceleration. We compute the JT expansion of the acceleration using either the JTTNP-k or the JTCNP-k method and we compute the difference between the values given by the JT expansion and the ones obtained by a Monte Carlo estimation (i.e. the ones obtained by evaluating for each sample the analytic expression of the perturbation under consideration), determining the average acceleration error using (35). Comparing the acceleration errors corresponding to all particular positions, the worst cases of the JT computations can be found, this is, the cases with the largest average error. Finally, the optimal expansion order is defined as the lowest order $k$ of JTTNP-k (or JTCNP-k) whose largest average acceleration error measured on a certain uncertainty neighborhood, satisfies the given accuracy threshold. In what follows only the results for these worst cases are displayed. 


\section{Solar radiation pressure perturbation}

The adopted SRP acceleration model is expressed in the ECI reference frame as [24]:

$$
\boldsymbol{a}_{S R P, E C I}=-v P C_{r} \frac{A}{m} \frac{\boldsymbol{r}_{\odot}}{r_{\odot}^{3}} A U^{2},
$$

where $\boldsymbol{r}_{\odot}$ indicates the Sun's position vector with respect to the spacecraft, $P$ is the solar radiation pressure at a distance of $1 \mathrm{AU}, v$ is the shadow function (its computation is also given in [24]). In our analysis we assume that the shape of the spacecraft is a sphere. The radiation pressure coefficient $C_{r}$, the mass $m$, and the cross-sectional area $A$ are related to the individual spacecraft properties.

In the propagations of the GEO model, the SRP acceleration must be converted into the LVLH reference frame by means of:

$$
a_{S R P, L V L H}=\mathcal{T}_{E C I}^{L V L H} \cdot a_{S R P, E C I} .
$$

To determine the optimal expansion order of the SRP acceleration, the epoch January 5 th, 2015, 0:0:0 UTC has been chosen, in which the Earth is very close to the perigee of its orbit; nevertheless, other relative positions provide similar results. Sampling the spacecraft position along the GEO region, we found that the worst case occurs when the Earth-Sun line and the projection of the spacecraft position vector on the ecliptic plane are aligned (see Figure 5). In this case, the Cartesian coordinates of the spacecraft, in the ECI reference frame, are $[11099.9,-40676.9,0.0] \mathrm{km}$.

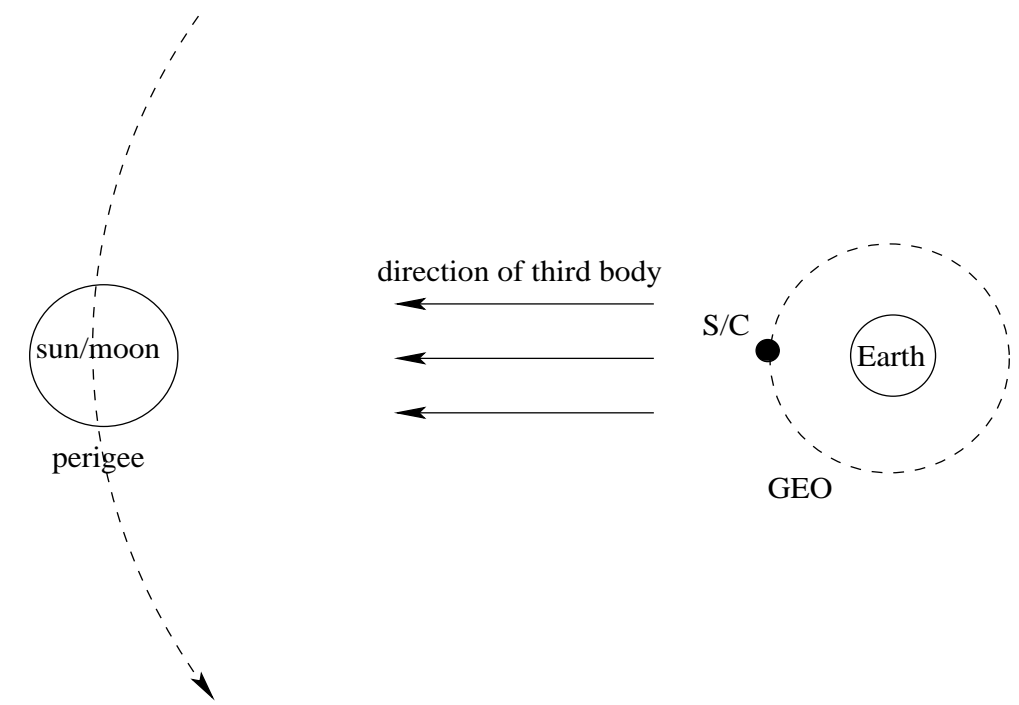

Fig. 5 Schematic diagram of the case where we display results for the SRP and luni-solar attracting accelerations.

We remark that in the computations, the ratio of the spacecraft's cross-sectional area and its mass are set equal to 1, and the radiation pressure coefficient $C_{r}$ is also set equal to 1 . With these values, the SRP acceleration acting on the center of the spacecraft is $4.72 \times 10^{-6} \mathrm{~m} / \mathrm{s}^{2}$. For a common GEO spacecraft, its area-to-mass ratio is approximately 
equal to 0.01 , and the radiation pressure coefficient $C_{r}$ is usually less than 1.5 , thus, the order of magnitude of SRP acceleration should be around $10^{-8} \mathrm{~m} / \mathrm{s}^{2}$. Therefore, as it was already discussed, it is reasonable to assume a value for accuracy threshold equal to $10^{-11} \mathrm{~m} / \mathrm{s}^{2}$, since it is three orders of magnitude smaller than the SRP acceleration. Moreover, it has to be mentioned that the Earth's shadow has also been considered in the simulations.

The uncertainty neighborhood for the simulation is defined as $\{x \pm \Delta x, y \pm \Delta y, z \pm \Delta z\}$, where $\Delta x=\Delta y=\Delta z=\Delta$. A multivariable uniform distribution has been used to take $1331\left(=11^{3}\right)$ samples inside the uncertainty neighborhood. Figure 6 illustrates the average SRP acceleration error using both JTTNP-k and JTCNP-k expansions, from which it follows that the average errors of both expansions are almost the same. Recall that the optimal expansion order is determined when the error satisfies the accuracy threshold condition. According to Figure 6, the SRP acceleration is almost constant when $\Delta \leq 215 \mathrm{~km}$. As a consequence, we can take for the SRP acceleration, on the central position of the uncertainty neighborhood, a constant value when $\Delta \leq 215 \mathrm{~km}$, while the first order expansion must be applied when $\Delta \in(215,300] \mathrm{km}$.

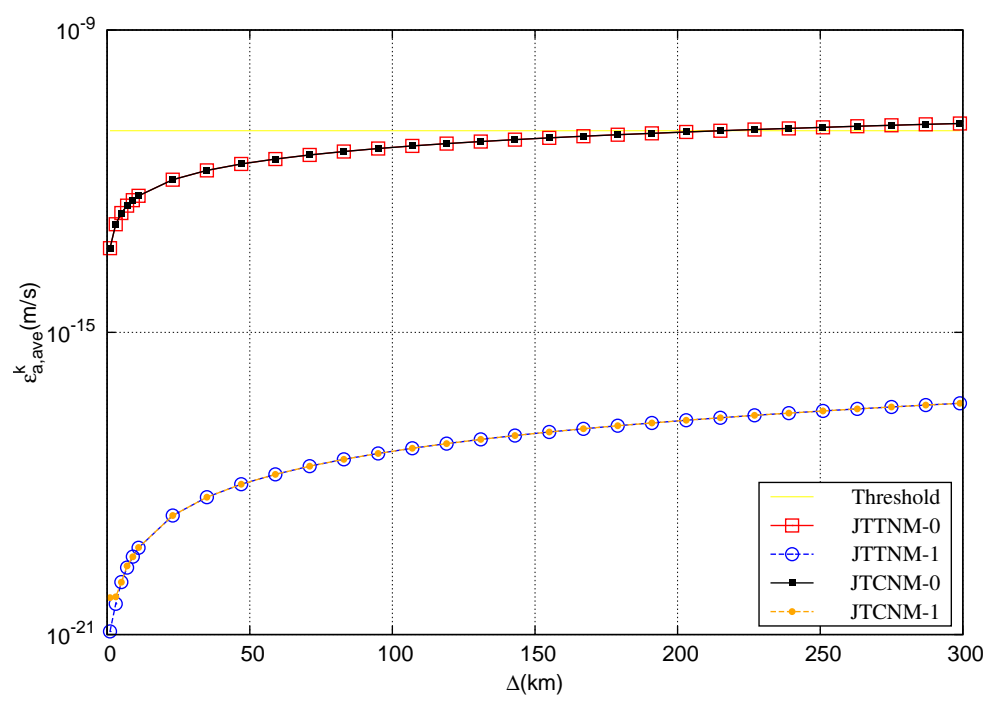

Fig. 6 Average SRP acceleration error using JTTNP-k and JTCNP-k expansions.

\section{Luni-solar gravitational perturbation}

The gravitational acceleration of a point mass $M$ (Moon or Sun) on the spacecraft can be expressed in the ECI reference frame as:

$$
\boldsymbol{a}_{M, E C I}=G M\left(\frac{\boldsymbol{r}_{M}-\boldsymbol{r}}{\left|\boldsymbol{r}_{M}-\boldsymbol{r}\right|^{3}}-\frac{\boldsymbol{r}_{M}}{\left|r_{M}\right|^{3}}\right),
$$

where $\boldsymbol{r}_{M}$ and $\boldsymbol{r}$ represent the Earth-centered coordinates of the mass $M$ and of the spacecraft, respectively. The position vector $\boldsymbol{r}_{M}$ can be computed by the method given in [24] or using any other kind of ephemerides. Again we note that when we propagate a trajectory using the GEO dynamic model, the acceleration must be converted into the 
LVLH reference frame by means of:

$$
\boldsymbol{a}_{M, L V L H}=\mathcal{T}_{E C I}^{L V L H} \cdot \boldsymbol{a}_{M, E C I}
$$

The epoch January 5th, 2015, 0:0:0 UTC, (spacecraft Geocentric position vector $[11099.9,-40676.9,0] \mathrm{km}$ ) has also been used for the computations of the solar perturbations. For other epochs the results are of the same order of magnitude. Figure $\square$ displays the average error, using both JTTNP-k and JTCNP-k expansions, for the solar perturbing acceleration. The average errors of both expansions are of the same order of magnitude, and it must also be noted that the JTTNP-1 and JTCNP-1 are accurate enough even for a big uncertainty neighborhood. So the optimal expansion order for the solar perturbation is taken equal to 1 .

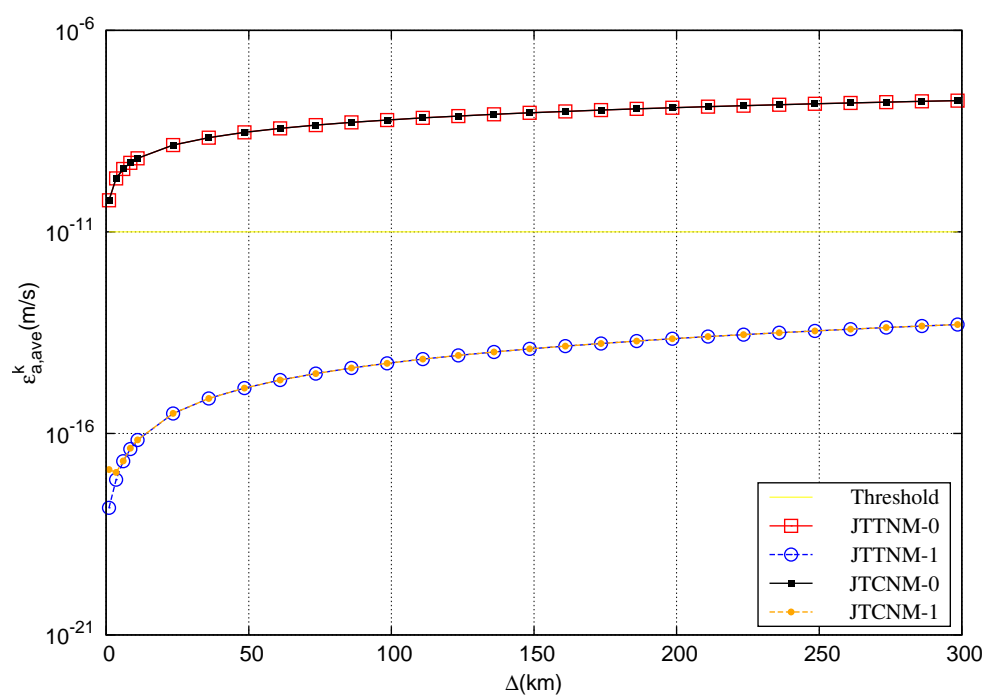

Fig. 7 Average acceleration error using JTTNP-k and JTCNP-k expansions for the approximation of the solar perturbing acceleration.

The same procedure is applied for the lunar perturbing acceleration case. The epoch is now January 21th, 2015, 21:36:0 UTC, in which the Moon is closest to the Earth (see Figure [1), but other epochs along a synodic month provide similar results. The spacecraft position is sampled in the GEO region and the worst case position is $[11099.9,-40676.9,0.0] \mathrm{km}$.

Figure 8 shows that there are no differences in the average errors between the JTTNP-k and JTCNP-k expansions. Note that the first order expansion is necessary to approximate the lunar perturbing acceleration when $\Delta \leq 140 \mathrm{~km}$; furthermore, when $\Delta \in(140,300] \mathrm{km}$, the optimal expansion order should be chosen as 2 .

\section{Earth's non-spherical gravity perturbations}

The Earth's potential has a significant impact on the evolution of spacecraft trajectories. For its computation, we have used the implementation given in [24] which is briefly summarized in what follows. In the ECEF reference frame, Earth's gravitational acceleration can be written as: 


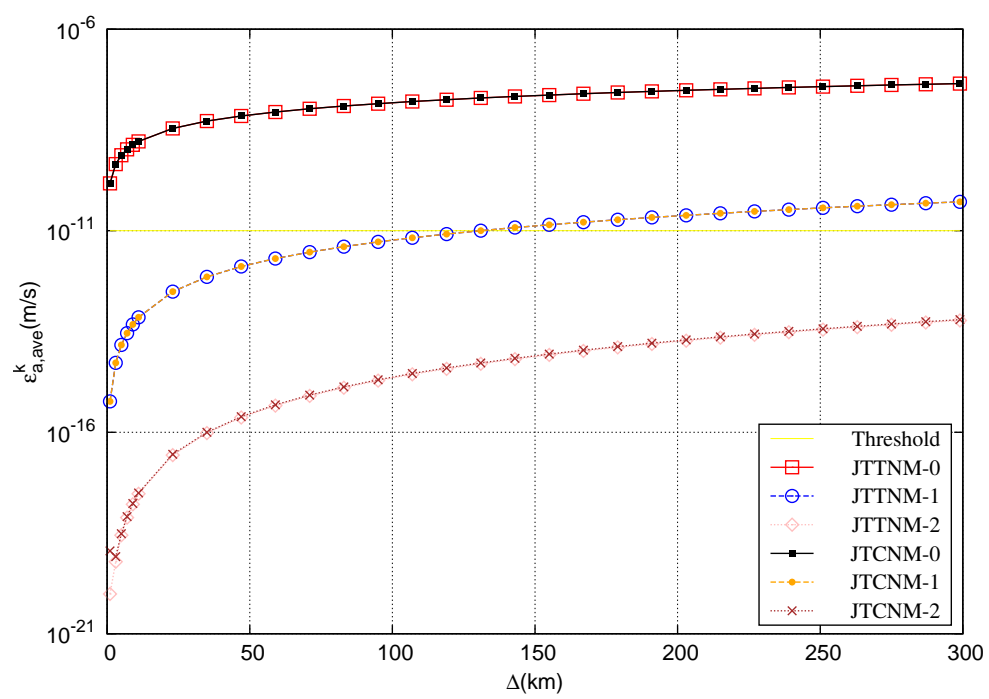

Fig. 8 Average acceleration error using JTTNP-k and JTCNP-k expansions for the approximation of the lunar perturbing acceleration.

$$
\boldsymbol{a}_{g, E C E F}=\left(\sum_{n, m} a_{x, n m}, \sum_{n, m} a_{y, n m}, \sum_{n, m} a_{z, n m}\right) .
$$

The components $a_{x, n m}, a_{y, n m}$, and $a_{z, n m}$ can be expressed as:

$$
\begin{aligned}
& a_{x, n m}=\frac{G M_{e}}{2 R_{\oplus}^{2}}\left\{\left(-C_{n m} V_{n+1, m+1}-S_{n m} W_{n+1, m+1}\right)+\frac{(n-m+2) !}{(n-m) !}\left(C_{n m} V_{n+1, m-1}+S_{n m} W_{n+1, m-1}\right)\right\}, \\
& a_{y, n m}=\frac{G M_{e}}{2 R_{\oplus}^{2}}\left\{\left(-C_{n m} W_{n+1, m+1}+S_{n m} V_{n+1, m+1}\right)+\frac{(n-m+2) !}{(n-m) !}\left(-C_{n m} W_{n+1, m-1}+S_{n m} V_{n+1, m-1}\right)\right\}, \\
& a_{z, n m}=\frac{G M_{e}}{R_{\oplus}^{2}}\left\{(n-m+1)\left(-C_{n m} V_{n+1, m}-S_{n m} W_{n+1, m}\right)\right\},
\end{aligned}
$$

where $G M_{e}$ is the gravitational coefficient of the Earth, $R_{\oplus}$ is the Earth's equatorial radius, and $C_{n m}, S_{n m}$ are the geopotential coefficients given by the Earth gravity model. In our work, a $5 \times 5$ gravity model of the EGM96S model has been adopted, that is, $0 \leq m \leq n \leq 5$. Note that $m=n=0$ corresponds to the gravitational acceleration of an ideal spherical Earth. The $V_{n m}$ and $W_{n m}$ values satisfy the recurrence relations:

$$
\begin{aligned}
V_{m m} & =(2 m-1)\left\{\frac{x R_{\oplus}}{r^{2}} V_{m-1, m-1}-\frac{y R_{\oplus}}{r^{2}} W_{m-1, m-1}\right\}, \\
W_{m m} & =(2 m-1)\left\{\frac{x R_{\oplus}}{r^{2}} W_{m-1, m-1}+\frac{y R_{\oplus}}{r^{2}} V_{m-1, m-1}\right\}, \\
V_{n m} & =\left(\frac{2 n-1}{n-m}\right) \frac{z R_{\oplus}}{r^{2}} V_{n-1, m}-\left(\frac{n+m-1}{n-m}\right) \frac{R_{\oplus}^{2}}{r^{2}} V_{n-2, m}, \\
W_{n m} & =\left(\frac{2 n-1}{n-m}\right) \frac{z R_{\oplus}}{r^{2}} W_{n-1, m}-\left(\frac{n+m-1}{n-m}\right) \frac{R_{\oplus}^{2}}{r^{2}} W_{n-2, m},
\end{aligned}
$$


where $r$ denotes the spacecraft distance with respect to the Earth's mass center. To compute $V_{n m}$ and $W_{n m}$, the following initial values are required:

$$
V_{00}=\frac{R_{\oplus}}{r}, \quad W_{00}=0, \quad V_{m-1, m}=0, \quad W_{m-1, m}=0
$$

Since the above formulae provide the Earth's gravitational acceleration in the ECEF reference frame, some coordinate transformations are required to obtain the resulting accelerations in the ECI or in the LVLH reference frames. The transformation matrix between the ECI and LVLH reference frames is given by (II2). The time-dependent linear transformation between the ECEF and ECI reference frames is given by the matrix:

$$
\mathcal{T}_{E C E F}^{E C I}=\left(\begin{array}{ccc}
\cos \left(\omega_{e} t\right) & -\sin \left(\omega_{e} t\right) & 0 \\
\sin \left(\omega_{e} t\right) & \cos \left(\omega_{e} t\right) & 0 \\
0 & 0 & 1
\end{array}\right),
$$

where $\omega_{e}=7.292115 \times 10^{-5} \mathrm{rad} / \mathrm{s}$ is the average Earth's rotational angular speed. Therefore, when the Cartesian dynamical model is used for the propagation of trajectories, the Earth's gravitational acceleration is expressed in the ECI reference frame as:

$$
\boldsymbol{a}_{g, E C I}=\mathcal{T}_{E C E F}^{E C I} \cdot \boldsymbol{a}_{g, E C E F}
$$

Otherwise, if the propagation is carried out in the GEO dynamic model, the acceleration should be converted into the LVLH reference frame by means of:

$$
\boldsymbol{a}_{g, L V L H}=\mathcal{T}_{E C I}^{L V H} \cdot \mathcal{T}_{E C E F}^{E C I} \cdot \boldsymbol{a}_{g, E C E F}
$$

where the matrices $\mathcal{T}_{E C I}^{L V L H}$ and $\mathcal{T}_{E C E F}^{E C I}$ are, respectively, given by (102) and (401).

To obtain the optimal expansion order for the Earth's perturbing gravitational acceleration (excluding the central gravitational acceleration), we first sample the spacecraft's position along the GEO region, with the purpose of finding the longitude where the perturbing acceleration varies in a most significative way over an unitary position deviation. It happens that the worst case corresponds to a longitude equal to $161^{\circ} \mathrm{E}$, and the uncertainty neighborhood that has been taken is around this critical position. The implementations of the nonlinear maps, using both JTTNP-k and JTCNP-k expansions, indicate that their average errors are again almost the same, as shown in Figure Q According to the results, when $\Delta \leq 21 \mathrm{~km}$, the optimal expansion order is 1 , when $21<\Delta \leq 185 \mathrm{~km}$, the optimal expansion order is 2 , and when $185<\Delta \leq 300 \mathrm{~km}$, the optimal expansion order is 3 . 


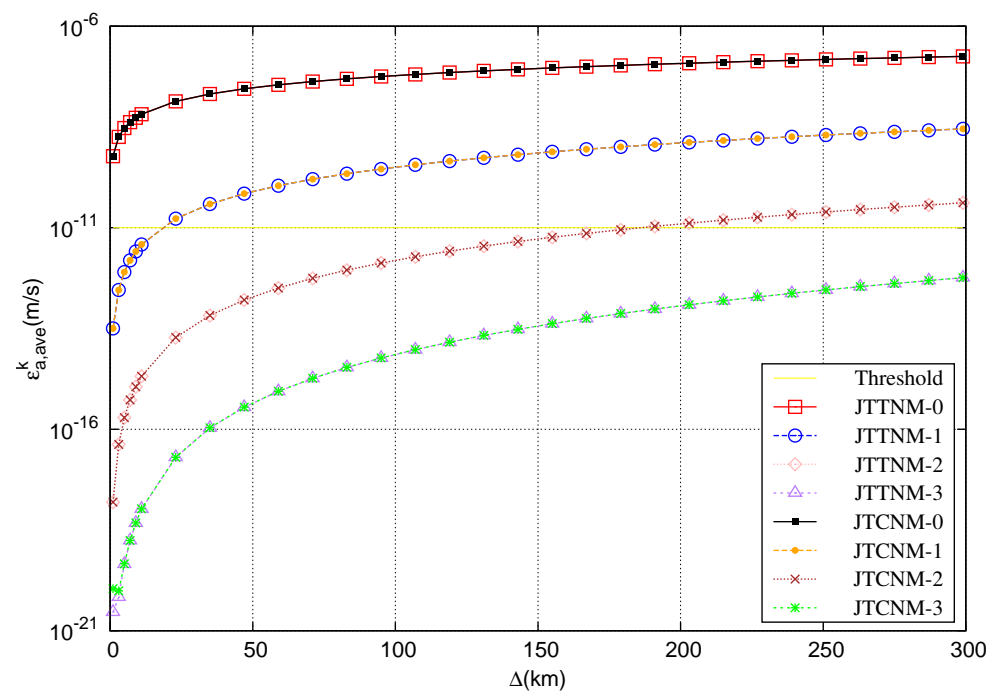

Fig. 9 Average acceleration error using JTTNP-k and JTCNP-k expansions to approximate the Earth's gravitational perturbations.

\section{JT-based orbit propagations in Cartesian and GEO models}

In the preceding section the optimal orders of the main orbital perturbations have been determined, for both the JTTNP-k and the JTCNP-k expansions. This is a key point for the accuracy and computational cost of the trajectory propagation. It has been shown that the average errors of the JTTNP-k and JTCNP-k methods are very similar, but the computational cost associated to the JTCNP-k is much higher than the one associated to the JTTNP-k expansions. Therefore, the JTTNP-k procedure is the one that has been used to propagate the geotrajectories. Nevertheless, the more computationally intensive JTCNP-k expansions yields essentially the same results, and we omit them for short.

Letting aside the influence of the evaluation of orbital perturbations, the performance of the uncertainty propagation still depends on more factors: the coordinate representation of the vectorfield, the size of uncertainty neighborhood, the order of the JT expansion, the numerical integrator and the total propagation time. It is also apparent that the accuracy decreases in accordance with the total integration time interval and the size of the uncertainty neighborhood, while the computational cost increases with the propagation time interval.

To compare the performance of JT-based implementations in the Cartesian and GEO models, we fix the propagation time to two days, and we choose a fixed uncertainty neighborhood. In addition, the slow variations of the vectorfield and of the perturbations in the GEO regime lead to a nearly fixed integration time step. Therefore, we consider fixed time step JTRK methods (JTRK4, JTRK8) to propagate geostationary trajectories. We note that both, the coordinate representation and the order of the integrator, impact on the integration time step which further influence the total computational cost. This performance comparison between Cartesian and GEO models leads to proposing some recommendations for propagating geostationary trajectory uncertainties. Of course, the optimal expansion orders for evaluating the perturbations discussed in the previous section are used in the computations. 
One of the key points of the analysis is the obtention of the "optimal" integration time step in each situation. This study is conducted in the Cartesian and GEO models involving the four dominant perturbing accelerations, and compares the pointwise Monte Carlo simulations (this is, the classical propagation of single trajectories with initial conditions inside the uncertainty neighborhood) with the results using JTTNP-k expansions. The pointwise Monte Carlo simulations are done very accurately integrating the GEO model using a variable-step 7-8 Runge-Kutta numerical integrator. Taking into account that JTTNP-k expansions have their worse accuracy at the border of the uncertainty neighborhood, 5000 uniformly distributed sampling points are taken on the surface of the initial state uncertainty sphere to evaluate the differences between the expansions and each accurate pointwise propagation.

Table 3 Average position error when propagating the Cartesian model using JTRK8

\begin{tabular}{ccccccccc}
\hline \multirow{2}{*}{$\Delta$} & $k$ & \multicolumn{7}{c}{ time step size } \\
& & $\leq 600 \mathrm{~s}$ & $1200 \mathrm{~s}$ & $1800 \mathrm{~s}$ & $2400 \mathrm{~s}$ & $3000 \mathrm{~s}$ & $3600 \mathrm{~s}$ & $4800 \mathrm{~s}$ \\
\hline \multirow{2}{*}{$\Delta r=1 \mathrm{~km}$} & $1 s t$ & 5.8257 & 5.8257 & 5.8244 & 5.8119 & 5.7540 & 6.0908 & 20.476 \\
& $2 n d$ & 0.0016 & 0.0016 & 0.0049 & 0.0519 & 0.3447 & 1.6351 & 19.450 \\
\hline$\Delta r=10 \mathrm{~km}$ & $1 s t$ & 582.56 & 582.56 & 582.56 & 582.55 & 582.47 & 582.14 & 578.16 \\
& $2 n d$ & 1.2973 & 1.2973 & 1.2974 & 1.3027 & 1.3919 & 2.1149 & 19.458 \\
& $3 r d$ & 0.0025 & 0.0025 & 0.0060 & 0.0526 & 0.3451 & 1.6356 & 19.452 \\
& $4 t h$ & 0.0006 & 0.0007 & 0.0046 & 0.0518 & 0.3447 & 1.6351 & 19.452 \\
\hline \multirow{2}{*}{$\Delta v=0.1 \mathrm{~m} / \mathrm{s}$} & $1 s t$ & 10.928 & 10.928 & 10.926 & 10.909 & 10.809 & 10.739 & 22.004 \\
& $2 n d$ & 0.0034 & 0.0034 & 0.0060 & 0.0519 & 0.3447 & 1.6351 & 19.450 \\
\hline$\Delta v=3 \mathrm{~km}$ & $1 s t$ & 6432.24 & 6432.24 & 6432.24 & 6817.77 & 6817.70 & 6817.44 & 6813.98 \\
& 2nd & 45.407 & 45.407 & 45.407 & 45.406 & 45.411 & 45.565 & 51.895 \\
& 3rd & 0.2404 & 0.2405 & 0.2431 & 0.2767 & 0.5263 & 1.7688 & 19.559 \\
& $4 t h$ & 0.0143 & 0.0143 & 0.0154 & 0.0538 & 0.3453 & 1.6357 & 19.454 \\
\hline
\end{tabular}

Note: $k$ indicates the computational order and $\Delta=(\Delta r, \Delta v)$ describes the uncertainty neighborhood. The simulation time is two days and the position error is given in meters. The order is cut when no further improvement is observed.

The initial conditions and spacecraft parameters used are given in Tables m. Four different initial uncertainty neighborhoods are considered. The first two ones take the sampling points uniformly distributed on the surface of uncertainty spheres of radius $\Delta r=1 \mathrm{~km}$ and $\Delta r=10 \mathrm{~km}$ about the nominal state (i.e. positions are sampled on the sphere while the velocity is kept equal to the one of the nominal state). The third case only considers uncertainties in the velocity components, $\Delta v=0.1 \mathrm{~m} / \mathrm{s}$, and the fourth case considers that all the state variables are affected by initial uncertainties, described by means of two spheres: $\Delta r=30 \mathrm{~km}$, and $\Delta v=1 \mathrm{~m} / \mathrm{s}$. Clearly, the uncertainty neighborhoods in the first and third cases are relative small while in the second and fourth cases are relatively big. It should be noted that the average position error depends not only on the truncation error of the JT Taylor expansion but also on the truncation error of the numerical integrator, due to the stepsize, and it is also limited by the accuracy of the numerical computations. 
Table 3 shows the average position error for the propagations in the Cartesian model using the JTRK8. As expected, the size of the uncertainty neighborhood plays an important role on the definition of the optimal computational order. The second order Taylor expansion JTTNP-2 is accurate enough to propagate the initial uncertainties in the first and third cases, while a third order JTTNP-3 is required to propagate a larger initial uncertainty neighborhoods, such as the ones of the second and fourth test cases. Note that, generally speaking, the "optimal" time step is only defined when the computational order is accurate enough, and the accuracy of the computational order is closely related with the size of the uncertainty neighborhood. This is, for a given uncertainty neighborhood, the computational order has to be high enough to provide accurate enough expansions. If the expansion representation of the neighborhood is not accurate enough, there is a point where decreasing the integration time step does not improve the error. The limiting error values obtained by reducing the time step are due to the numerical rounding errors when the JT expansion is accurate enough, while when the JT expansion is not accurate enough, the accuracy limit is dominated by the inaccuracy of the JT expansion. In other words, for a given uncertainty neighborhood, the dominating errors using a high order JTTNP-k and a small integration step are mainly rounding errors of the numerical computations. On the contrary, for low orders JTTNP-k and short integrations steps, the errors mainly come form the truncation of relevant terms of the JTTNP-k.

Table 4 Average position error when propagating the GEO model using JTRK8

\begin{tabular}{cccccccccc}
\hline \multirow{2}{*}{$\Delta$} & $k$ & $6 \mathrm{ks}$ & $8 \mathrm{ks}$ & $10 \mathrm{ks}$ & $13 \mathrm{ks}$ & $15 \mathrm{ks}$ & $18 \mathrm{ks}$ & $21 \mathrm{ks}$ & $24 \mathrm{ks}$ \\
\hline \multirow{2}{*}{$\Delta r=1 \mathrm{~km}$} & $1 s t$ & 0.1521 & 0.1522 & 0.1528 & 0.1576 & 0.1722 & 0.2947 & 0.2592 & 2.6314 \\
& $2 n d$ & 0.0001 & 0.0002 & 0.0008 & 0.0059 & 0.0240 & 0.1433 & 0.3153 & 2.4795 \\
& $3 r d$ & 0.00009 & 0.00018 & 0.0008 & 0.0059 & 0.0240 & 0.1433 & 0.3153 & 2.4795 \\
\hline \multirow{2}{*}{$\Delta r=10 \mathrm{~km}$} & $1 s t$ & 15.213 & 15.213 & 15.214 & 15.218 & 15.230 & 15.346 & 15.024 & 17.680 \\
& $2 n d$ & 0.1028 & 0.1028 & 0.1029 & 0.1034 & 0.1087 & 0.1704 & 0.3331 & 2.4795 \\
& $3 r d$ & 0.0010 & 0.0011 & 0.0013 & 0.0063 & 0.0244 & 0.1437 & 0.3150 & 2.4800 \\
& $4 t h$ & 0.0010 & 0.0010 & 0.0012 & 0.0059 & 0.0240 & 0.1432 & 0.3154 & 2.4794 \\
\hline \multirow{2}{*}{$\Delta v=0.1 \mathrm{~m} / \mathrm{s}$} & $1 s t$ & 0.8252 & 0.8250 & 0.8244 & 0.8201 & 0.8103 & 0.7296 & 1.0965 & 1.6597 \\
& $2 n d$ & 0.0003 & 0.0004 & 0.0008 & 0.0059 & 0.0240 & 0.1433 & 0.3154 & 2.4793 \\
$\Delta v=1 \mathrm{~m} / \mathrm{s}$ & 2nd & 0.0001 & 0.0002 & 0.0008 & 0.0059 & 0.0240 & 0.1433 & 0.3154 & 2.4793 \\
\hline & 3rd & 0.0682 & 0.0683 & 0.0688 & 0.0735 & 0.0892 & 0.2104 & 0.2865 & 2.5434 \\
& $4 t h$ & 0.0112 & 0.0112 & 0.0113 & 0.0136 & 0.0256 & 0.1440 & 0.3182 & 2.4766 \\
\hline
\end{tabular}

Note: $k$ indicates the computational order and $\Delta=(\Delta r, \Delta v)$ describes the uncertainty neighborhood. The simulation time is two days and the position error is given in meters. The order is cut when no further improvement is observed.

The same simulations have also been done replacing the Cartesian model with the GEO model; the corresponding results are given in Table 4. Comparing this results with the ones of Table B, it follows that for propagating trajectories in the GEO model we can take a larger optimal time step size, $13000 \mathrm{~s} \approx 3.6 \mathrm{~h}$, for a position final accuracy threshold 
Table 5 Average position error when propagating the Cartesian model using JTRK4

\begin{tabular}{cccccccc}
\hline \multirow{2}{*}{$\Delta$} & $k$ & \multicolumn{7}{c}{ time step size } \\
& & $\leq 100 \mathrm{~s}$ & $200 \mathrm{~s}$ & $300 \mathrm{~s}$ & $400 \mathrm{~s}$ & $500 \mathrm{~s}$ & $600 \mathrm{~s}$ \\
\hline \multirow{2}{*}{$\Delta r=1 \mathrm{~km}$} & $1 s t$ & 5.8260 & 5.8324 & 5.8653 & 5.9824 & 6.3362 & 7.2769 \\
& $2 n d$ & 0.0021 & 0.0231 & 0.1333 & 0.4758 & 1.2963 & 2.9815 \\
& $3 r d$ & 0.0016 & 0.0231 & 0.1333 & 0.4758 & 1.2963 & 2.9815 \\
\hline \multirow{2}{*}{$\Delta r=10 \mathrm{~km}$} & $1 s t$ & 582.56 & 582.57 & 582.60 & 582.70 & 582.92 & 583.37 \\
& $2 n d$ & 1.2973 & 1.2993 & 1.3251 & 1.4561 & 1.9035 & 3.1472 \\
& $3 r d$ & 0.0028 & 0.0227 & 0.1327 & 0.4753 & 1.2958 & 2.9810 \\
\hline$\Delta v=0.1 \mathrm{~m} / \mathrm{s}$ & $1 s t$ & 10.928 & 10.937 & 10.980 & 11.121 & 11.501 & 12.419 \\
& $2 n d$ & 0.0038 & 0.0230 & 0.1331 & 0.4757 & 1.2962 & 2.9814 \\
& $3 r d$ & 0.0012 & 0.0230 & 0.1332 & 0.4758 & 1.2964 & 2.9815 \\
\hline$\Delta r=30 \mathrm{~km}$ & $1 s t$ & 6432.24 & 6432.25 & 6432.28 & 6432.37 & 6432.58 & 6433.01 \\
$\Delta v=1 \mathrm{~m} / \mathrm{s}$ & $2 n d$ & 45.4074 & 45.4077 & 45.4104 & 45.4330 & 45.5463 & 45.9080 \\
& 3rd & 0.2399 & 0.2368 & 0.2673 & 0.4973 & 1.2412 & 2.8974 \\
& $4 t h$ & 0.0144 & 0.0274 & 0.1334 & 0.4756 & 1.2961 & 2.9813 \\
\hline
\end{tabular}

Note: $k$ indicates the computational order and $\Delta=(\Delta r, \Delta v)$ describes the uncertainty neighborhood. The simulation time is two days and the position error is given in meters. The order is cut when no further improvement is observed.

Table 6 Average position error when propagating the GEO model using JTRK4

\begin{tabular}{ccccccccc}
\hline \multirow{2}{*}{$\Delta$} & $k$ & $900 \mathrm{~s}$ & $1200 \mathrm{~s}$ & $1800 \mathrm{~s}$ & $2400 \mathrm{~s}$ & $4800 \mathrm{~s}$ & $7200 \mathrm{~s}$ & $9600 \mathrm{~s}$ \\
\hline \multirow{2}{*}{$\Delta r=1 \mathrm{~km}$} & $1 s t$ & 0.1520 & 0.1518 & 0.1507 & 0.1485 & 0.1354 & 0.3041 & 1.6709 \\
& $2 n d$ & 0.0002 & 0.0004 & 0.0016 & 0.0043 & 0.0246 & 0.1521 & 1.5188 \\
& $3 r d$ & 0.0001 & 0.0003 & 0.0016 & 0.0043 & 0.0246 & 0.1521 & 1.5189 \\
\hline \multirow{2}{*}{$\Delta r=10 \mathrm{~km}$} & $1 s t$ & 15.213 & 15.212 & 15.211 & 15.209 & 15.191 & 15.355 & 16.721 \\
& $2 n d$ & 0.1028 & 0.1028 & 0.1029 & 0.1031 & 0.1076 & 0.1754 & 1.5184 \\
& $3 r d$ & 0.0010 & 0.0010 & 0.0016 & 0.0038 & 0.0241 & 0.1526 & 1.5189 \\
& $4 t h$ & 0.0010 & 0.0010 & 0.0016 & 0.0043 & 0.0246 & 0.1521 & 1.5184 \\
\hline \multirow{2}{*}{$\Delta v=0.1 \mathrm{~m} / \mathrm{s}$} & $1 s t$ & 0.8253 & 0.8255 & 0.8267 & 0.8295 & 0.8498 & 0.7231 & 0.9024 \\
& $2 n d$ & 0.0003 & 0.0005 & 0.0016 & 0.0043 & 0.0246 & 0.1521 & 1.5189 \\
& $3 r d$ & 0.0001 & 0.0003 & 0.0016 & 0.0043 & 0.0246 & 0.1521 & 1.5189 \\
\hline \multirow{2}{*}{$\Delta r=30 \mathrm{~km}$} & $1 s t$ & 161.07 & 161.07 & 161.07 & 161.07 & 161.07 & 161.10 & 161.40 \\
& $2 n d$ & 6.5975 & 6.5975 & 6.5975 & 6.5975 & 6.5980 & 6.6082 & 7.0398 \\
& $3 r d$ & 0.0681 & 0.0680 & 0.0674 & 0.0665 & 0.0650 & 0.2200 & 1.5823 \\
& $4 t h$ & 0.0112 & 0.0112 & 0.0115 & 0.0125 & 0.0267 & 0.1533 & 1.5155 \\
\hline$N=1 \mathrm{~m} / \mathrm{s}$
\end{tabular}

Note: $k$ indicates the computational order and $\Delta=(\Delta r, \Delta v)$ describes the uncertainty neighborhood. The simulation time is two days and the position error is given in meters. The order is cut when no further improvement is observed. 
of $\varepsilon_{r, \max }=2 \mathrm{~cm}$.

In addition to using the fixed-step JTRK8, the JTRK4 is another choice for propagations in the Cartesian and GEO models and we present the resulting average position errors in Tables $\square$ and 6 . We find that the optimal time step for propagations in the Cartesian model with the JTRK4 integrator is $100 \mathrm{~s}$ for a position accuracy threshold of $\varepsilon_{r, \max }=2 \mathrm{~cm}$. Similarly, from Table 6 follows that a time step of $2400 \mathrm{~s}$ is optimal to integrate the GEO dynamic with a JTRK4 integrator with the same accuracy threshold.

In the preceding tables (from Table [ $]$ to Table 6 ) we have a variety of cases with the aim of exploring the impact of coordinate representations, integrator, computational order, and uncertainty neighborhood on the performance of JTTNP-k expansions for propagating geotrajectories. It is interesting to remark that the choices of the coordinate representation and the integrator do not influence the accuracy of the JTTNP-k method, however, they have a joint effect on the optimal time step and on the total computational cost. On the other hand, these results show that the influence of the size of the uncertainty neighborhood and the computational order on the optimal time step is almost negligible. The accuracy of the JTTNP-k plays the major role in the scene. In what follows, a fairly good position accuracy threshold, $\varepsilon_{r, \max }=2 \mathrm{~cm}$, and a poor position accuracy one, $\varepsilon_{r, \max }=40 \mathrm{~cm}$ will be assumed. Based on the previous experiences, Table $\square$ gives some recommendations on the optimal time step for the propagations. This table includes also results for one day and one week propagations. Note that there are slight variations on the optimal time step depending on the length of the propagation time, mostly due to the accumulated global truncation errors of the integrations. As was previously stated, the main point of divergence of the JT methods is that, since the size of the transported neighborhood increases with time, the expansion can not achieve the required accuracy without domain splitting techniques (see [18, [19]) and in this case the optimal step size of the integrator is not defined. This is indicated with a "*” in Table 廿.

Table 7 Recommendation on the optimal time step size (unit: s)

\begin{tabular}{cccccccc}
\hline \multirow{2}{*}{ Model } & \multirow{2}{*}{ Integrator } & \multicolumn{3}{c}{$\epsilon \leq 2 \mathrm{~cm}$} & \multicolumn{3}{c}{$\epsilon \leq 40 \mathrm{~cm}$} \\
\cline { 3 - 8 } & & $\mathrm{t}=1$ & $\mathrm{t}=2$ & $\mathrm{t}=7$ & $\mathrm{t}=1$ & $\mathrm{t}=2$ & $\mathrm{t}=7$ \\
\hline \multirow{2}{*}{ Cartesian } & JTRK4 & 200 & 100 & $100^{*}$ & 400 & 300 & $200^{*}$ \\
& JTRK8 & 2400 & 1800 & $1200^{*}$ & 3000 & 3000 & $2400^{*}$ \\
\multirow{2}{*}{ GEO } & JTRK4 & 4800 & 2400 & $2400^{*}$ & 7200 & 7200 & $4800^{*}$ \\
& JTRK8 & 13000 & 13000 & $13000^{*}$ & 21000 & 21000 & $15000^{*}$ \\
\hline
\end{tabular}

Note: Optimal time step size refers to the maximum time step size satisfying the accuracy constraint $\varepsilon$ for $t$ equal to one, two and seven days propagations. The upperscript $*$ indicates that the optimal time step size is only defined in the small uncertainty neighborhood cases, this is, in the first and third cases, since for the other cases the JT expansion is not accurate enough.

Furthermore, the computational cost, using a JTTNP-k method together with the recommended optimal time step for two days propagations of geotrajectories, has been also studied. Table 8 displays the computational time spent by 
Table 8 Computational time (s) for 2 days propagation

\begin{tabular}{ccccc}
\hline \multirow{2}{*}{ Model } & \multirow{2}{*}{ Integrator } & \multicolumn{3}{c}{ Computational order } \\
\cline { 3 - 5 } & & 2nd & 3rd & 4th \\
\hline \multirow{2}{*}{ Cartesian } & JTRK4(100 s) & 262.1 & 282.5 & 297.9 \\
& JTRK8(1800 s) & 30.6 & 32.7 & 33.5 \\
\multirow{2}{*}{ GEO } & JTRK4(2400 s) & 11.2 & 11.9 & 13.5 \\
& JTRK8(13000s $)$ & 4.7 & 5.1 & 5.7 \\
\hline
\end{tabular}

Table 9 Recommendation of optimal computational order

\begin{tabular}{|c|c|c|c|c|c|c|c|c|c|}
\hline \multirow{3}{*}{ Model } & \multirow{3}{*}{ Integrator } & \multicolumn{8}{|c|}{$(\delta r, \delta v)$} \\
\hline & & \multicolumn{2}{|c|}{$(1 \mathrm{~km}, 0)$} & \multicolumn{2}{|c|}{$(10 \mathrm{~km}, 0)$} & \multicolumn{2}{|c|}{$(0,0.1 \mathrm{~m} / \mathrm{s})$} & \multicolumn{2}{|c|}{$(30 \mathrm{~km}, 1 \mathrm{~m} / \mathrm{s})$} \\
\hline & & $\epsilon \leq 2$ & $\epsilon \leq 40$ & $\epsilon \leq 2$ & $\epsilon \leq 40$ & $\epsilon \leq 2$ & $\epsilon \leq 40$ & $\epsilon \leq 2$ & $\epsilon \leq 40$ \\
\hline \multirow{2}{*}{ Cartesian } & JTRK4 & $(2,2,3)$ & $(2,2,2)$ & $(3,3, *)$ & $(2,3,4)$ & $(2,2,3)$ & $(2,2,2)$ & $(3,4, *)$ & $(3,3, *)$ \\
\hline & JTRK8 & $(2,2,3)$ & $(2,2,2)$ & $(3,3, *)$ & $(2,3, *)$ & $(2,2,3)$ & $(2,2,2)$ & $(3,4, *)$ & $(3,3, *)$ \\
\hline \multirow{2}{*}{ GEO } & JTRK4 & $(2,2,2)$ & $(1,1,2)$ & $(3,3, *)$ & $(2,2,3)$ & $(2,2,2)$ & $(1,2,2)$ & $(3,4, *)$ & $(3,3, *)$ \\
\hline & JTRK8 & $(2,2,2)$ & $(1,1,2)$ & $(3,3, *)$ & $(2,2,3)$ & $(2,2,2)$ & $(1,2,2)$ & $(3,4, *)$ & $(3,3, *)$ \\
\hline
\end{tabular}

Note: Optimal computational order refers to the minimum order satisfying the accuracy constraint $\varepsilon$ (in $\mathrm{cm}$ ) for one, two, and seven days propagations, $\left(k_{1}, k_{2}, k_{7}\right)$. A value $*$ indicates that it is impossible to satisfy the required accuracy using any high order expansion.

a laptop processor Intel(R) Core(TM) i5-7300HQ provided with GNU-Linux C++ compiler. Clearly, the propagations of GEO dynamic model carried out using the JTRK8 integrator are much faster than other cases, with a substantial reduction in the computational cost. So, the comparative between JTRK4 and JTRK8 shows that the latter behaves better in the aspect of computational burden. Analogously, the usage of the GEO model also reduces the computational cost. Moreover, for the orders that one could need, the computational order plays a slight impact on the computational time, as shown in Table 8 .

Finally, note also that the average position errors in Table 1 are smaller than the ones in Table B, when the same computational order is considered. Sometimes there are also some additional advantages when propagating using the GEO model, since a lower order can be suitable enough. For example, to satisfy the position accuracy threshold, $\varepsilon_{r, \max }=40 \mathrm{~cm}$, a third order expansion is necessary when the propagation is done in the Cartesian model, while a second order expansion is accurate enough using the GEO model. Table 9 shows this fact, as well as other valuable recommendations on the optimal computational order.

\section{Conclusions}

Using Jet Transport methods, this manuscript studies geostationary trajectory uncertainty propagation subjected to the four dominant perturbations. The achieved results correspond to several different simulations using different coordinate representations, integrator, computational order, and uncertainty neighborhoods. Both Chebyshev and 
Taylor expansions are considered in the JT-based schemes.

We conclude that the average errors of both methodologies are of the same order of magnitude, but the JTTNP-k method, based on Taylor expansions, is much faster than the JTCNP-k method, based on Chebyshev expansions. This disadvantage of the JTCNP-k method becomes more apparent when the computational order and the number of variables increases, to the point that it significantly limits the practical application of the JTCNP-k method, albeit it possesses a more uniform error distribution. This problem would become more apparent for a large dimension ODE system, or when the expansions include the uncertainty of many parameters impacting the trajectory, or the orbit determination. Furthermore, the implementation of the JTTNP-k method shows that it is an efficient and accurate method for geotrajectory uncertainty propagations under a highly perturbed vectorfield.

To further save computational cost, this work explores the optimal expansion order for approximating the dominant perturbing accelerations, as well as the study of the most suitable coordinate representation and integrator. It has been demonstrated that the GEO model behaves better than the Cartesian model, and generally, a JTRK8 improves the computational efficiency. As for the choice of the computational order, one must take into account the accuracy threshold and the size of the uncertainty neighborhood. To simplify the procedure, some valuable tables are given to provide recommendations for a future study of the GEO orbit determination, suitable for new applications demanding higher accuracies and low computational burden.

\section{Appendix}

\section{Relation between GEO elements and Cartesian coordinates}

This appendix gives a detailed derivation of the explicit relation between GEO elements and Cartesian coordinates. In the derivation process, the Keplerian element set $\{a, e, i, \omega, \Omega, \theta\}$ is used as a bridge. The spacecraft position and velocity vectors can be expressed in the perifocal reference frame as:

$$
\begin{aligned}
& \boldsymbol{r}=(r \cos \theta, r \sin \theta, 0)^{T}, \\
& \boldsymbol{v}=\left(-\frac{\mu}{h} \sin \theta, \frac{\mu}{h}(e+\cos \theta), 0\right)^{T}
\end{aligned}
$$

The transformation matrix between the ECI and the perifocal reference frames can be computed using Keplerian element set, this is:

$$
\mathcal{T}_{\text {peri }}^{E C I}=\left(\begin{array}{ccc}
\cos \Omega \cos \omega-\sin \Omega \sin \omega \cos i & -\cos \Omega \sin \omega-\sin \Omega \cos \omega \cos i & \sin \Omega \sin i \\
\sin \Omega \cos \omega+\cos \Omega \sin \omega \cos i & -\sin \Omega \sin \omega+\cos \Omega \cos \omega \cos i & -\cos \Omega \sin i \\
\sin \omega \sin i & \cos \omega \sin i & \cos i
\end{array}\right)
$$


According to (1), we obtain,

$$
\begin{gathered}
\sin \Omega=\frac{Q_{1}}{\sqrt{Q_{1}^{2}+Q_{2}^{2}}}, \quad \cos \Omega=\frac{Q_{2}}{\sqrt{Q_{1}^{2}+Q_{2}^{2}}}, \quad 1-\cos i=\frac{2\left(Q_{1}^{2}+Q_{2}^{2}\right)}{1+Q_{1}^{2}+Q_{2}^{2}}, \\
\cos ^{2} \Omega+\sin ^{2} \Omega \cos i=\frac{1-Q_{1}^{2}+Q_{2}^{2}}{1+Q_{1}^{2}+Q_{2}^{2}}, \quad \sin ^{2} \Omega+\cos ^{2} \Omega \cos i=\frac{1+Q_{1}^{2}-Q_{2}^{2}}{1+Q_{1}^{2}+Q_{2}^{2}},
\end{gathered}
$$

and using (8) we have, $\omega+\theta=s-\Omega$.

Based on the above equations, the spacecraft position and velocity vectors can be transformed into the ECI reference frame by means of the transformation matrix $\mathcal{T}_{\text {peri }}^{E C I}$. For instance, the $x$ component of the spacecraft's position vector is given by:

$$
\begin{aligned}
x & =r \cdot[\cos \Omega \cos (\omega+\theta)-\sin \Omega \sin (\omega+\theta) \cos i] \\
& =r \cdot\left(\cos ^{2} \Omega \cos s+\sin \Omega \cos \Omega \sin s-\sin \Omega \cos \Omega \sin s \cos i+\sin ^{2} \Omega \cos s \cos i\right) \\
& =r \cdot\left[\cos \Omega \sin \Omega \sin s(1-\cos i)+\cos s\left(\cos ^{2} \Omega+\sin ^{2} \Omega \cos i\right)\right] \\
& =\frac{r}{1+Q_{1}^{2}+Q_{2}^{2}}\left[2 Q_{1} Q_{2} \sin s+\left(1-Q_{1}^{2}+Q_{2}^{2}\right) \cos s\right],
\end{aligned}
$$

where $r$ is given by $(\mathbb{Z})$, and $s$ is calculated by means of (지). The same procedure is carried out to compute the other components of the spacecraft position and velocity vectors, which are the following explicit functions of the GEO elements:

$$
\begin{aligned}
x & =\frac{r}{1+Q_{1}^{2}+Q_{2}^{2}}\left[2 Q_{1} Q_{2} \sin s+\left(1-Q_{1}^{2}+Q_{2}^{2}\right) \cos s\right], \\
y & =\frac{r}{1+Q_{1}^{2}+Q_{2}^{2}}\left[2 Q_{1} Q_{2} \cos s+\left(1+Q_{1}^{2}-Q_{2}^{2}\right) \sin s\right], \\
z & =\frac{2 r}{1+Q_{1}^{2}+Q_{2}^{2}}\left[Q_{2} \sin s-Q_{1} \cos s\right], \\
\dot{x} & =\frac{\mu}{h\left(1+Q_{1}^{2}+Q_{2}^{2}\right)}\left[2 Q_{1} Q_{2}\left(\cos s+e_{x}\right)+\left(-1+Q_{1}^{2}-Q_{2}^{2}\right)\left(\sin s+e_{y}\right)\right], \\
\dot{y} & =\frac{\mu}{h\left(1+Q_{1}^{2}+Q_{2}^{2}\right)}\left[-2 Q_{1} Q_{2}\left(\sin s+e_{y}\right)+\left(1+Q_{1}^{2}-Q_{2}^{2}\right)\left(\cos s+e_{x}\right)\right], \\
\dot{z} & =\frac{\mu}{h\left(1+Q_{1}^{2}+Q_{2}^{2}\right)}\left[Q_{1}\left(\sin s+e_{y}\right)+Q_{2}\left(\cos s+e_{x}\right)\right] .
\end{aligned}
$$

\section{Computations of matrices $A$ and $B$}

The explicit relationship between Cartesian coordinates and GEO elements can be written as:

$$
x_{c}=g\left(x_{G}, t\right)
$$


where $\boldsymbol{x}_{c}, \boldsymbol{x}_{G}$, respectively, represent the Cartesian coordinate set and the GEO element set. According to the chain rule, taking the full derivative of (43) with respect to time $t$ gives:

$$
\dot{\boldsymbol{x}}_{c}=\boldsymbol{A}\left(\boldsymbol{x}_{G}, t\right) \dot{x}_{G}+\boldsymbol{B}\left(\boldsymbol{x}_{G}, t\right)
$$

where $\boldsymbol{A}\left(\boldsymbol{x}_{G}, t\right)=\partial \boldsymbol{g} / \partial \boldsymbol{x}_{G}$, and $\boldsymbol{B}\left(\boldsymbol{x}_{G}, t\right)=\partial \boldsymbol{g} / \partial t$ can be computed explicitly from ([1]), this is:

$$
\begin{aligned}
& \frac{\partial g_{1}}{\partial \lambda}=\frac{r^{2} \dot{x}}{h}, \quad \frac{\partial g_{2}}{\partial \lambda}=\frac{r^{2} \dot{y}}{h}, \quad \frac{\partial g_{3}}{\partial \lambda}=2 r \cdot\left[\frac{Q_{2} \cos s+Q_{1} \sin s}{1+Q_{1}^{2}+Q_{2}^{2}}+\frac{z}{2 p}\left(e_{x} \sin s-e_{y} \cos s\right)\right] \\
& \frac{\partial g_{4}}{\partial \lambda}=-\frac{\mu x}{h r}, \quad \frac{\partial g_{5}}{\partial \lambda}=-\frac{\mu y}{h r}, \quad \frac{\partial g_{6}}{\partial \lambda}=-\frac{\mu z}{h r}, \quad \frac{\partial g_{1}}{\partial \delta \bar{a}}=\frac{x}{1+\delta \bar{a}}, \quad \frac{\partial g_{2}}{\partial \delta \bar{a}}=\frac{y}{1+\delta \bar{a}}, \quad \frac{\partial g_{3}}{\partial \delta \bar{a}}=\frac{z}{1+\delta \bar{a}} \\
& \frac{\partial g_{4}}{\partial \delta \bar{a}}=-\frac{\dot{x}}{2(1+\delta \bar{a})}, \quad \frac{\partial g_{5}}{\partial \delta \bar{a}}=-\frac{\dot{y}}{2(1+\delta \bar{a})}, \quad \frac{\partial g_{6}}{\partial \delta \bar{a}}=-\frac{\dot{z}}{2(1+\delta \bar{a})}, \quad \frac{\partial g_{1}}{\partial e_{x}}=-\frac{2 x e_{x}}{1-e_{x}^{2}-e_{y}^{2}}-\frac{x r \cos s}{p} \\
& \frac{\partial g_{2}}{\partial e_{x}}=-\frac{2 y e_{x}}{1-e_{x}^{2}-e_{y}^{2}}-\frac{y r \cos s}{p}, \quad \frac{\partial g_{3}}{\partial e_{x}}=-\frac{2 z e_{x}}{1-e_{x}^{2}-e_{y}^{2}}-\frac{z r \cos s}{p}, \quad \frac{\partial g_{4}}{\partial e_{x}}=\frac{2 \mu Q_{1} Q_{2}}{h\left(1+Q_{1}^{2}+Q_{2}^{2}\right)}+\frac{\dot{x} e_{x}}{1-e_{x}^{2}-e_{y}^{2}} \\
& \frac{\partial g_{5}}{\partial e_{x}}=\frac{\mu\left(1+Q_{1}^{2}-Q_{2}^{2}\right)}{h\left(1+Q_{1}^{2}+Q_{2}^{2}\right)}+\frac{\dot{y} e_{x}}{1-e_{x}^{2}-e_{y}^{2}}, \quad \frac{\partial g_{6}}{\partial e_{x}}=\frac{2 \mu Q_{2}}{h\left(1+Q_{1}^{2}+Q_{2}^{2}\right)}+\frac{\dot{z} e_{x}}{1-e_{x}^{2}-e_{y}^{2}}, \quad \frac{\partial g_{1}}{\partial e_{y}}=-\frac{2 x e_{x}}{1-e_{x}^{2}-e_{y}^{2}}-\frac{x r \sin s}{p} \\
& \frac{\partial g_{2}}{\partial e_{y}}=-\frac{2 y e_{x}}{1-e_{x}^{2}-e_{y}^{2}}-\frac{y r \sin s}{p}, \quad \frac{\partial g_{3}}{\partial e_{y}}=-\frac{2 z e_{x}}{1-e_{x}^{2}-e_{y}^{2}}-\frac{z r \sin s}{p}, \quad \frac{\partial g_{4}}{\partial e_{y}}=-\frac{\mu\left(1-Q_{1}^{2}+Q_{2}^{2}\right)}{h\left(1+Q_{1}^{2}+Q_{2}^{2}\right)}+\frac{\dot{x} e_{y}}{1-e_{x}^{2}-e_{y}^{2}} \\
& \frac{\partial g_{5}}{\partial e_{y}}=-\frac{2 \mu Q_{1} Q_{2}}{h\left(1+Q_{1}^{2}+Q_{2}^{2}\right)}+\frac{\dot{y} e_{y}}{1-e_{x}^{2}-e_{y}^{2}}, \quad \frac{\partial g_{6}}{\partial e_{y}}=\frac{2 \mu Q_{1}}{h\left(1+Q_{1}^{2}+Q_{2}^{2}\right)}+\frac{\dot{z} e_{y}}{1-e_{x}^{2}-e_{y}^{2}}, \quad \frac{\partial g_{1}}{\partial Q_{1}}=z-\frac{2 x Q_{1}}{1+Q_{1}^{2}+Q_{2}^{2}} \\
& \frac{\partial g_{2}}{\partial Q_{1}}=\frac{2 r\left(Q_{1} \sin s+Q_{2} \cos s\right)-2 Q_{1} y}{1+Q_{1}^{2}+Q_{2}^{2}}, \quad \frac{\partial g_{3}}{\partial Q_{1}}=-\frac{2\left(z Q_{1}+r \cos s\right)}{1+Q_{1}^{2}+Q_{2}^{2}}, \quad \frac{\partial g_{4}}{\partial Q_{1}}=\dot{z}-\frac{2 \dot{x} Q_{1}}{1+Q_{1}^{2}+Q_{2}^{2}} \\
& \frac{\partial g_{5}}{\partial Q_{1}}=\frac{2 \mu\left(Q_{1} e_{x}-Q_{2} e_{y}\right)}{h\left(1+Q_{1}^{2}+Q_{2}^{2}\right)}+\frac{\partial g_{6}}{\partial \lambda}-\frac{2 Q_{1} \dot{y}}{1+Q_{1}^{2}+Q_{2}^{2}}, \quad \frac{\partial g_{6}}{\partial Q_{1}}=\frac{2 \mu\left(e_{y}+\sin s\right)}{h\left(1+Q_{1}^{2}+Q_{2}^{2}\right)}-\frac{2 Q_{1} \dot{z}}{1+Q_{1}^{2}+Q_{2}^{2}} \\
& \frac{\partial g_{1}}{\partial Q_{2}}=\frac{2 r\left(Q_{1} \sin s+Q_{2} \cos s\right)-2 Q_{2} x}{1+Q_{1}^{2}+Q_{2}^{2}}, \frac{\partial g_{2}}{\partial Q_{2}}=-z-\frac{2 y Q_{2}}{1+Q_{1}^{2}+Q_{2}^{2}}, \quad \frac{\partial g_{3}}{\partial Q_{2}}=-\frac{-2\left(z Q_{2}-r \sin s\right)}{1+Q_{1}^{2}+Q_{2}^{2}} \\
& \frac{\partial g_{4}}{\partial Q_{2}}=\frac{2 \mu\left(Q_{1} e_{x}-Q_{2} e_{y}\right)}{h\left(1+Q_{1}^{2}+Q_{2}^{2}\right)}+\frac{\partial g_{6}}{\partial \lambda}-\frac{2 Q_{2} \dot{x}}{1+Q_{1}^{2}+Q_{2}^{2}}, \quad \frac{\partial g_{5}}{\partial Q_{2}}=-\dot{z}-\frac{2 \dot{y} Q_{2}}{1+Q_{1}^{2}+Q_{2}^{2}}, \frac{\partial g_{6}}{\partial Q_{2}}=\frac{2 \mu\left(e_{x}+\cos s\right)}{h\left(1+Q_{1}^{2}+Q_{2}^{2}\right)}-\frac{2 Q_{2} \dot{z}}{1+Q_{1}^{2}+Q_{2}^{2}}
\end{aligned}
$$

Besides,

$$
\frac{\partial g_{i}}{\partial t}=\omega_{e} \cdot \frac{\partial g_{i}}{\partial \lambda} \quad i=1 \ldots 6
$$

\section{Acknowledgments}

J.C. thanks the support of Doctorate Foundation of Northwestern Polytechnical University and the Chinese Scholarship Council. He also thanks National natural science foundation of China for the grant 11572248, MINECO- FEDER 
for the grant MTM2015-65715-P and Key Laboratory of Aerospace Flight Dynamics for the grant 2015afd1015. J.J.M. thanks MINECO- FEDER for the grant MTM2015-65715-P and the Catalan government for the grant 2017SGR1049. G.G. thanks the Catalan grant government for the grant 2017SGR-1374, and MINECO-FEDER for the grant MTM2016-80117-P.

\section{References}

[1] Lemmens, S., “Classification of Geosynchronous Objects,” Tech. Rep. Issue 19, European Space Agency, April, 2017.

[2] IADC, "Support to the IADC Space Debris Mitigation Guidelines,” Tech. rep., Inter-Agency Debris Committee, 2014. IADC04-06.

[3] Fuster, R. M., Usón, M. F., and Ibars, A. B., "Interferometric orbit determination for geostationary satellites," Science China Information Sciences, Vol. 60, No. 6, 2017, p. 060302. doi:10.1007/s11432-016-9052-y.

[4] Ribo, S., Arco, J. C., Cardellach, E., Oliveras, S., Rius, A., and Buck, C., "Preliminary results of digital satellite TV opportunity signals scattered on the sea-surface," Workshop on Reflectometry using GNSS and Other Signals of Opportunity (GNSS+R), Purdue University, West Lafayette, USA, October 10-11, 2012.

[5] Soop, E. M., Handbook of geostationary orbits, Kluwer Academic, Dordrecht, The Netherlands, 1994, Chap. 2.3.

[6] Tombasco, J., "Orbit estimation of geosynchronous objects via ground-based and space-based optical tracking," Ph.D. thesis, University of Colorado at Boulder, 2011.

[7] Tombasco, J., Axelrad, P., and Jah, M., "Specialized coordinate representation for dynamic modeling and orbit estimation of geosynchronous orbits," Journal of guidance, control, and dynamics, Vol. 33, No. 6, 2010, pp. 1824-1836. doi:10.2514/1. 48903.

[8] Crassidis, J. L., and Junkins, J. L., Optimal estimation of dynamic systems, CRC press, Boca Raton, FL, 2004, pp. $243-401$.

[9] Maybeck, P. S., Stochastic models, estimation, and control, Academic press, New York, 1982, pp. 159-271.

[10] Park, R. S., and Scheeres, D. J., "Nonlinear mapping of Gaussian statistics: theory and applications to spacecraft trajectory design,” Journal of guidance, Control, and Dynamics, Vol. 29, No. 6, 2006, pp. 1367-1375. doi:10.2514/1.20177.

[11] Berz, M., Modern map methods in particle beam physics, Vol. 108, Academic Press, 1999.

[12] Valli, M., Armellin, R., di Lizia, P., and Lavagna, M. R., "Nonlinear mapping of uncertainties in celestial mechanics,” Journal of Guidance, Control, and Dynamics, Vol. 36, No. 1, 2012, pp. 48-63. doi:10.2514/1.58068.

[13] Cavenago, F., Di Lizia, P., Massari, M., Servadio, S., and Wittig, A., "DA-based nonlinear filters for spacecraft relative state estimation,” 2018 Space Flight Mechanics Meeting, 2018, p. 1964. 
[14] Valli, M., Armellin, R., di Lizia, P., and Lavagna, M. R., "Nonlinear filtering methods for spacecraft navigation based on differential algebra," Acta Astronautica, Vol. 94, No. 1, 2014, pp. 363 - 374. doi:10.1016/j.actaastro.2013.03.009.

[15] Armellin, R., Di Lizia, P., and Zanetti, R., "Dealing with uncertainties in angles-only initial orbit determination," Celestial Mechanics and Dynamical Astronomy, Vol. 125, No. 4, 2016, pp. 435-450. doi:10.1007/s10569-016-9694-z.

[16] Wittig, A., and Armellin, R., "High order transfer maps for perturbed Keplerian motion," Celestial Mechanics and Dynamical Astronomy, Vol. 122, No. 4, 2015, pp. 333-358. doi:10.1007/s10569-015-9621-8.

[17] Palau, D. P., "Dynamical transport mechanisms in celestial mechanics and astrodynamics problems," Ph.D. thesis, Universitat de Barcelona, 2016.

[18] Wittig, A., Di Lizia, P., Armellin, R., Makino, K., Bernelli-Zazzera, F., and Berz, M., "Propagation of large uncertainty sets in orbital dynamics by automatic domain splitting," Celestial Mechanics and Dynamical Astronomy, Vol. 122, No. 3, 2015, pp. 239-261. doi:10.1007/s10569-015-9618-3.

[19] Pérez-Palau, D., Gómez, G., and Masdemont, J. J., "A new subdivision algorithm for the flow propagation using polynomial algebras," Communications in Nonlinear Science and Numerical Simulation, Vol. 61, 2018, pp. 37-53. doi:10.1016/j.cnsns. 2018.01.013.

[20] Riccardi, A., Tardioli, C., and Vasile, M., "An intrusive approach to uncertainty propagation in orbital mechanics based on Tchebycheff polynomial algebra," Proceedings of the AAS/AIAA Astrodynamics Specialist Conference, American Astronautical Society, Vail, Colorado, August 2015.

[21] Ortega Absil, C., Riccardi, A., Vasile, M., and Tardioli, C., "SMART-UQ: uncertainty quantification toolbox for generalised intrusive and non intrusive polynomial algebra," 6th International Conference on Astrodynamics Tools and Techniques, 2016.

[22] “DACE Developmet Group, DA core engine 2.0,” https://github.com/dacelib/dace, 2017.

[23] Isserlis, L., "On a formula for the product-moment coefficient of any order of a normal frequency distribution in any number of variables,” Biometrika, Vol. 12, No. 1/2, 1918, pp. 134-139. doi:10.2307/2331932.

[24] Montenbruck, O., and Gill, E., Satellite orbits: models, methods and applications, Springer Science \& Business Media, 2012. 